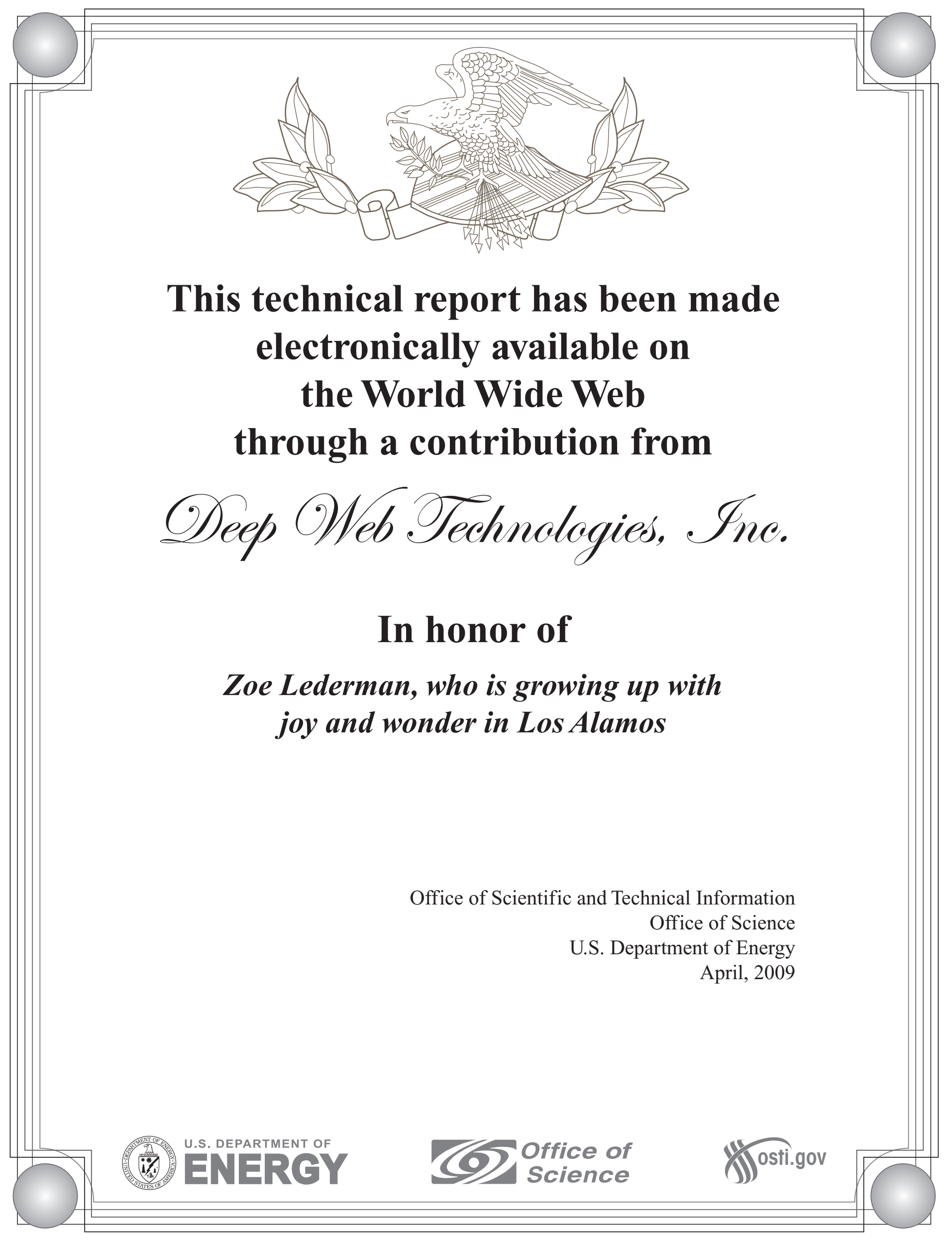


ENGINEERING RESEARCH INSTITUTE

\section{DEVI.ATIONS FROM THERMAL EQUILIBRIUM IN SHOCK WAVES}

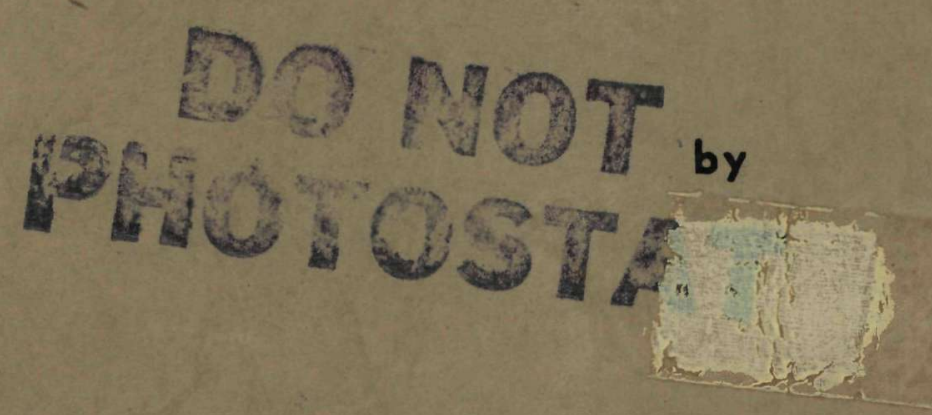

H. A. BETHE

E. TELLER

UNIVERSITY OF MICHIGAN • ANN ARBOR $665-001$ 


\section{DISCLAIMER}

This report was prepared as an account of work sponsored by an agency of the United States Government. Neither the United States Government nor any agency Thereof, nor any of their employees, makes any warranty, express or implied, or assumes any legal liability or responsibility for the accuracy, completeness, or usefulness of any information, apparatus, product, or process disclosed, or represents that its use would not infringe privately owned rights. Reference herein to any specific commercial product, process, or service by trade name, trademark, manufacturer, or otherwise does not necessarily constitute or imply its endorsement, recommendation, or favoring by the United States Government or any agency thereof. The views and opinions of authors expressed herein do not necessarily state or reflect those of the United States Government or any agency thereof. 


\section{DISCLAIMER}

Portions of this document may be illegible in electronic image products. Images are produced from the best available original document. 
DEVIATIONS FRON THERMAL EQUILIBRIUM IN SHOCK WAVES

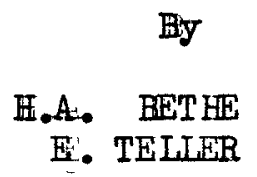

This document is

PUBLICLY RELEASABLE

hanh Kinuw

Authorizilig Óficial

Date: $476 / 09$ 


\section{NOTE}

This paper was written by Professor H. A. Bethe of Cornell University and Professor E. Teller of George Washington University before they had any official connections with Government agencies during World War II. Because of the importance of the paper, the Balih Ilstic Research Laboratories, Aberdeen Proving Ground, Aberdeen, Maryland, undertook the reproduction of this paper for limited distribution. This was done with the permission of the authors.

Since copies of this first reproduction are unavaliable, the Engineering Research Institute of the University of Michigan has obtained the permission of Professor Bethe to reproduce this paper. 
Deviations from Thermal Equilibrium in Shock Waves

Report by H. A. Bethe, Comear University, Ithaca, N. Y. In collaboration with (sections 1 and 2)

E. Teller, George Washington University, Washington, D. C.

\section{Contents}

\section{Page}

SUMMARY

NOTATIONS

1. The Energy Content of Gases ,

2. The Approach of Equilibrium Between Various Degrees of Freedom of the Molecules

A. Translation and Rotation

26

B. Vibration

C. Dissociation

D. Conclusions on the Excitation of Air

3. Theory of Shock Waves in the Case of Variable Specific Heat

4. The Approach of Equilibrium

5. Diffuse Shock Waves

61

6. The Temperature on the High Pressure Side 


\section{TABLES}

Page

I. Energy Content of a Harmonic Oscillator 10

II. Energy Content and Dissociation of Nitrogen and Oxygen 16

III. Energy Content and Dissociation of Air 19

IV. Theoretical Temperature Dependence of the Number of Collisions Necessary for De-excitation of the First Vibrational state

V. Experimental Results on the Excitation of Molecular Vibrations

VI. Relaxation Time and Mean Free Path of Vibration for $\mathrm{O}_{2}$ and $\mathrm{N}_{2}$ in $\mathrm{A}$ ir

VII. Relaxation Time and Mean Free Path for the Dissociation of Air

VIII. Characteristics of Shock Waves in Air 
DEVIATIONS FROM THERMAL EQUILIBRIUM IN SHOCK WAVES

\section{STMMARY}

The various terms contributing to the energy content of a gas, viz. translation, rotation, vibration, electronic excitation and dissociation, are discussed (par. 1). Tables are given of the energy content and the specific heat of a simple harmonic oscillator (Table I), of the energy content and the dissociation of nitrogen, oxygen (Table II) and air (Table III). The molecular vibration becomes important for air at about $600^{\circ} \mathrm{K}$, the dissociation at about $3000^{\circ} \mathrm{K}$.

The theory of shock waves is generalized to the case when the speclfic heat changes with temperature (par, 3). General formulae are obtained for the velocity, (3.10), density (3.8a), pressure (3.8b), and temperature (3.9) on the high pressure side. It 1s shown that the asymptotic values of $v_{2} \rho_{2} p$ and T on the high pressure side at sufficient distance from the front of the shock wave are uniquely determined by the values of these quantities on the low pressure side, and are independent of any intervening phenomeno connected with the approach of statistical equilibrium between the various degrees of freedom of the molecule. This we consider the most important result of the present investigations.

A table is given (Table VIII) of the asymptotic values of $v, p, p$ and $T$ on the high pressure side as a function of the velocity $v$ of the shock 
wave. The four quantities are calculated (a) using the actual specific heat of air as a function of temperature, as given by Table III (quantities are subscript in Table VIII), and (b) using a constant specific heat equal to that at $300^{\circ} \mathrm{K}$ (subscript 2). Large differences are found for the temperature in the two calculations $\left(\mathrm{T}_{2}=8000^{\circ}\right.$ when $\mathrm{T}_{3}=5000^{\circ}$ ) and for the density $\left(\rho_{2}=5.8, \rho_{3}=9.1\right)$ but the pressure, which 18 the most important quantity for applications, is almost independent of the specific heat $\left(p_{2}=155, p_{3}=168\right.$ atmospheres in the example quoted).

The sudden change of the temperature of a gas when passing through a shock wave destroys temporarily the statistical equilibrium between the various forms of energy of the gas molecules. The degrees of freedom of a molecule can conveniently be divided into two classes, the active ones and the inert ones (par. 2). The "active" degrees of freedom are translation and rotation; they come into thermal equilibrium after one or a few collisions (par. 2A). The most important "inert" degree of freedom is the vibration (par. 2B). Experiments on the absorption of sound show that between 20 and more than 500,000 collisions are necessary to establish thermal equilibrium between vibrations and the active degrees of freedom at room temperature (Table V). This result agrees with the expectation from the theory of Iandau and Teller. This theory allows one to predict with moderate accuracy the temperature dependence of $\mathrm{Z}$, the number of collistons necessary to de-excite the first vibrational quantum state, when a measurement of $\mathrm{Z}$ at one temperature is available (Table IV). Unfortunately no accurate measurements have been made for axygen and none at all for nitrogen, which makes quantitative statements almost impossible. Therefore we have only listed (Table VI) the values of the mean free path for vibration, $\lambda_{v}$ which follow from various assumption (par. 2D) about the efficiency of the collisions. The resulting values of $\lambda_{\nabla}$ for the cases which have practical importance for shock waves lie between 3 and 0.0016 millimeters, depending on the vibrating 
molecule $\left(\mathbb{N}_{2}\right.$ or $\left.\mathrm{O}_{2}\right)$, the essumption made about the efficlency of various molecular collisions, and the humidity of the air.

The dissociation also requires a considerable time to come into equilibrium. The theory (par. 2C) is somewhat more definite in this case than for the vibrations because it depends on the Boltzmann factor rather than on assumptions about the kinetics of collisions. Very large values (from 1 millimeter to 1 meter) are obtained (par. $2 D$, Table VII) for the mean free path for dissociation, $\lambda_{d}$, in practical cases.

The theory of par. 2 is applied to shock wave in par. 4 . It is shown that the shock wave has a sharp front on the low pressure side while on the high pressure side it extends over a distance of the order of the mean free path for vibration, $\lambda_{v}$, or for dissociation, $\lambda_{d}$. Immediately at the wave front, but on the high pressure side, the physical quantities $P, \rho, T, V$ can be calculated assuming that only the active degrees of freedom exist (Table VIII, quantities with subscripts 2). Going away from the wave front into the high pressure region, the energy $E_{i}$ of the inert degrees of freedom increases gradually and approaches the value corresponding to thermal equilibrium. In simple cases, $E_{1}, p, p, T$ and $v$ will follow an exponential law (cf. 4.10, 11). If the shock wave is violent enough so that dissociation occurs on the high pressure side, there is first a region of extension $\lambda_{v}$ near the wave front in which vibrational equilibrium is established but in which the dissociation is hardy affected, and then a much more extended region (extension $\lambda_{\alpha}$ ) in which dissociation takes place.

Two spectal cases of very soft shock waves are discussed in pars. 5 and 6, these discussions having mostly academic interest. In par. 5,we consider the case where the velocity of the shock wave $\nabla_{1}$ 1s between the actual velocity of sound $a_{1}$ and the sound velocity obtained by considering only the active degrees of freedom, i.e., 


$$
a_{1}<\nabla_{1}<a_{1}\left(1+\frac{\left(c_{v}-c_{v a}\right) R}{2 c_{v}-c_{v a}}\right)
$$

where $c_{v}$ is the total specific heat at constant volume and $c_{v a}$ the specific heat due to the active degrees of freedom only. It is show that in this narrow velocity interval the shock wave is diffuse on the low pressure as well as on the high pressure side. These diffuse shock waves go over automatically into shock waves with a sharp front when $\nabla_{1}$ approeches the upper limit given in (A).

In par. 6, slightly faster shock waves are considered, viz. waves for which

$$
a_{1}\left(I+\frac{\left(c_{v}-c_{v a}\right) R}{2 c_{v} c_{v a}} ;<v_{I}<a_{1}\left(I+\frac{R}{2^{c} v+R}\right)\right.
$$

For such waves, the temperature on the high pressure side increases with increasing distance from the wave front while for waves of higher velocity $v_{1}$, the temperature has its maximum immediately at the wave front and decreases from there. 
NOTATION

Throughout this Report, the following notations will be used:

$\rho=$ density of gas

$\mathrm{p}=$ pressure (in atmospheres)

$\mathrm{T}=$ temperature in degrees Kelvin

$\mathrm{E}=$ energy content of one gram of gas

$\alpha=$ degree of dissociation,

$1+\alpha=p /(\rho \mathrm{RT})$

$\beta=\frac{E}{p / \rho}+1$

$c_{p}=$ specific heat at constant pressure per gram

$c_{V}=$ specific heat at constant volume per gram

$\gamma=c_{p} / c_{\nabla}$

$\mathrm{R}=$ gas constant per gram

$R_{0}=$ gas constant per mol

$k=$ Boltzmann's constant (gas constant per molecule)

$\mathrm{h}=$ Planck's constant

$v=$ velocity of the gas

$m=\rho v=f l o w$ in grams per $\mathrm{cm}^{2}$ per sec

$\nabla=v+\frac{p}{m}$

c = velocity which the ges would have if streaming into vacuum

a $=$ velocity of sound

$\lambda_{\mathrm{V}}=$ mean free path for vibrations

$\lambda_{d}=$ mean free path for dissociation

$\nu=$ frequency of molecular vibrations

$D=$ dissociation energy of molecules 


\section{SUBSCRIPTS}

a for active degrees of freedom

i for inert degrees of freedom

$v$ for vibration

d for dissociation

h for an arbitrary point on the high pressure side of a shock wave

$\ell$ for an arbitrary point on the low pressure side

1 for a point on the low pressure side where thermal equilibrium exists between all degrees of freedom of the molecules

2 for a point on the high pressure side immediately at the front of the shock wave

3 for a point on the high pressure side at sufficient distance from the wave front so that thermal equilibrium exists 
Par. 1. The Energy Content of Cases

Perfect gases obey the equation of state

$$
p / p=R I
$$

where $p, p, T$ are pressure, density and absolute temperature and where the gas constant $R$ is a characteristic of the gas considered. If the gas dissociates, (1.I) ceases to be valid; in the particular case of a diatomic gas dissociating into atoms, we have instead:

$$
\mathrm{p} / \mathrm{p}=\operatorname{RT}(1+\alpha)
$$

where $\propto$ is the degree of dissoclation, i.e., the fraction of molecules dissociated. Generally, $\mathrm{p} / \mathrm{\rho} \mathrm{T}$ is proportional to the number of separate particles (molecules or atoms) per gram of the substance.

The energy content of a gas consists of five main parts, viz:

(1) the kinetic energy of the translation of the molecules

(2) the energy of molecular rotation

(3) the energy of Fibration

(4) the energy of electronic excitation of the molecule

(5) the energy of dissociation into atoms (or smaller groups of atoms).

We shall write the total energy content per gram in the form

$$
E=(\beta-1)(p / \rho)
$$

The inclusion of the term -I is convenient because the quantity occurring in the theory of shock waves is $E+p / \rho$, where $p / p$ is connected with the work done by the pressure. Furthermore, we shall denote by $\beta_{t}, \beta_{r}, \beta_{\mathrm{Y}}, \beta_{e}, \beta_{\mathrm{d}}$ the energy of translation, rotation, vibration, excitation, and dissociation, each divided by $p / \rho$, so that 


$$
\beta=\beta_{t}+\beta_{r}+\beta_{v}+\beta_{e}+\beta_{d}+1
$$

The varlous contributions will now be discussed in order:

1. The translational energy is $3 / 2 \mathrm{p} / \mathrm{\rho}$ for any gas, independent of the number of atoms per molecule, the temperature, etc.

2. The rotational energy, for all gases except $\mathrm{H}_{2}$, and at all temperatures at which the substance is gaseous, is given by the classical kinetic theory of gases without any important quantum correction. It is zero for atoms, I RT per gram for diatomic molecules and all polyatomic ones whose atams lie on a straight line such as $\mathrm{CO}_{2}$, and $3 / 2$ RT for all other polyatomic molecules. At high temperatures, there is a correction because the molecules change their shape due to their vibrations. This correction, cormonly called the interaction of vibrations and rotations, is usually not very great.

3. The vibrational energy can be approximated (at not too high temperatures) by resolving the vibration into normal modes and treating each mode as a harmonic oscillator.

The number of normal modes is 1 for a diatomic molecules, $3 n-5$ for a molecule containing $n$ atoms on a straight line and $3 n-6$ for a molecule with n atoms not on a straight line. The energy contained in one mode is (per gram)

$$
E_{V}=R T \frac{z}{e^{z}-I}
$$

where

$$
z=\frac{h \nu}{k^{T}}
$$

$\boldsymbol{V}$ is the frequency of the vibation, $h$ Planck's and $k$ Boltzmann's constant. If $\nu$ is given in wave numbers $\left(\mathrm{cm}^{-1}\right)$ and $\mathrm{T}$ in degrees Kelvin,

$$
z=1.438 \nu / T
$$


The frequencies of the various normal modes of molecules can be obtained from band spectra. A good survey of data can be found in H. Sponer, Molekilspektren I (Springer 1935). The frequencies of simple light molecules are very high, e.g. for $\mathrm{N}_{2}$ we have $\nu=2345 \mathrm{~cm}^{-1}$, for $\mathrm{O}_{2}, 1570 \mathrm{~cm}^{-1}$. For more complicated, and especially for polyatomic molecules, the lowest frequencies are much smaller, the highest ones of the same order as for diatomic ones. E.g., $\mathrm{CO}_{2}$ has four modes of vibration with frequencies $\nu=667,667,1336$ and $2350 \mathrm{~cm}^{-1}$.

For low temperatures ( 2 large), the vibrational energy is nerligible. E.g., for $z=5$, 1 t amounts only to $0.034 \mathrm{RT}, 1 . e$. I per cent of the value of $\mathrm{E}+\mathrm{p} / \rho$ for translation and rotation of a diatomic molecule. $z=5$ corresponds to $680^{\circ} \mathrm{K}$ for $\mathrm{N}_{2}, 450^{\circ}$ for $\mathrm{O}_{2}$ but only $192^{\circ}$ for the low frequency mode of $\mathrm{CO}_{2}$.

- Thus the vibrations mey be neglected at room temperature for $\mathrm{N}_{2}$ and $\mathrm{O}_{2}$ but not for $\mathrm{CO}_{2}$.

At high temperatures ( $\mathrm{z}$ omall), the vibrational energy is RT per mode. This value is attained very slowly. 


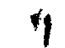

TABLE I

Energy Content of Harmonic Oscillator

$\begin{array}{llll}\frac{1}{\mathrm{z}}=\frac{\mathrm{kT}}{\mathrm{h} \nu} & \frac{E_{\mathrm{V}}}{\mathrm{RT}} & \frac{\mathrm{c}_{\mathrm{V}}}{\mathrm{R}} & \gamma \\ 0.05 & 4.1 \cdot 10^{-8} & 8.2 \cdot 10^{-7} & 1.400 \\ 0.1 & 4.5 \cdot 10^{-4} & 4.5 \cdot 10^{-3} & 1.399_{5} \\ 0.15 & 0.0085 & 0.0566 & 1.391 \\ 0.2 & 0.0339 & 0.170_{5} & 1.375 \\ 0.25 & 0.0747 & 0.304 & 1.357 \\ 0.3 & 0.123 & 0.426 & 1.342 \\ 0.4 & 0.223 & 0.609 & 1.321_{5} \\ 0.5 & 0.313 & 0.723 & 1.310_{5} \\ 0.6 & 0.389 & 0.800 & 1.303 \\ 0.8 & 0.502 & 0.880 & 1.296 \\ 1.0 & 0.582 & 0.921 & 1.292_{5} \\ 1.5 & 0.704 & 0.963 & 1.289 \\ 2 & 0.771 & 0.979 & 1.287_{5} \\ 1 \mathrm{arge} & 1-\frac{1}{2} \mathrm{z}+\frac{1}{12} \mathrm{z}^{2} & 1-\frac{1}{12} \mathrm{z}^{2} & 1.2857 \\ & & & \end{array}$


In Table I, we give the vibrational energy of a harmonic oscillator as a function of the temperature. We also give the contribution of the vibration to the specific heat, viz.

$$
c_{\nabla^{\prime} v}=\frac{D E_{v}}{\mathrm{dT}}
$$

which is seen to become appreclable at much smaller temperatures than $\mathbb{E}_{\mathrm{v}}$, and to approach its asymptotic value much faster. We have also included the ratio, $\gamma$ of the specific heats at constant pressure and volume, for a diatomic gas with harmonic oscillation, viz.

$$
\gamma=\frac{7 / 2 R+c_{v / v}}{5 / 2 R+c_{v} v}
$$

At hlgh temperatures, the vibrations can no longer be regarded as harmonic. The effect of the anharmonicity is to increase the energy content of the gas. E.8., for $\mathrm{N}_{2}$ at $5000^{\circ} \mathrm{K}$ the harmonic oscillator model would give $\beta_{v}=0.699$ while the correct value is 0.753 , including the interaction of vibration and rotation, and a small contribution from excitation. The influence of the anharmonicity is greater for molecules which are easily dissociated.

4. The electronic excitation is usually rather unimportant compared with vibration and dissociation.

5. The dissociation becomes important at temperatures above $2-3000^{\circ} \mathrm{K}$. If $\propto$ is the degree of dissociation, $p$ the total pressure, $p_{A}$ and $p_{M}$ the partial pressures of atoms and (diatomic) molecules, we have

$$
\frac{p_{A}^{2}}{p_{M}}=\frac{4 \alpha^{2} p}{1-\alpha^{2}}=K=e^{\Delta T / R_{O} T}
$$

* $p$ in (1.7) is considered a dimensionless quantity, viz. the ratio of the pressure to one atmosphere. 
The first equality follows from $p_{A}=\frac{2 \alpha}{1+\alpha} p, p_{M}=\frac{1-\alpha}{1+\alpha} p . \quad K$ is the dissociation constant, $R_{0}$ the gas constant per mole $=1.987$ calories $/$ degree, $\Delta F=F_{M}-F_{A}$ the difference of the free energies per mol of the molecular and the atomic gas, each taken at unit pressure. For some gases, Iike. $N_{2}$ and $\mathrm{O}_{2}$, tables of $\Delta \mathrm{F}$ as a function of temperature have been published (cf. below). Where they are not published, $\Delta F$ can be calculated from the Stern-Tetrode formula, which reads for diatomic geses

$$
K=\frac{\left(2 \pi m_{A} k T\right)^{3 / 2}}{n h^{3}} \cdot \frac{g_{A}^{2}}{G_{M}} e^{-\frac{D}{R_{0} T}}
$$

where $m_{A}$ is the mass of one atom, $n$ the number of separate particles per $\mathrm{cm}^{3}$ of the gas at unit pressure and temperature $T$, and $D$ the dissociation energy in calories per mol. According to spectroscopic evidence (Sponer), D has the value $117,200 \mathrm{cal}$. for oxygen and $182,000 \mathrm{cal}$. for nitrogen, so that $D / R_{0}=59,000$ and 91,600 degrees, respectively.

$8_{A}$ and $G_{M}$ are the statistical weights of the atom and the molecule. In general, $B_{A}$ may be put equal to the combined weight of all states of the multiplet to which the ground state belongs, so that

$$
\ddot{g}_{A}=(2 L+I)(2 S+I)
$$

where $I$ and $S$ are orbital angular momentum and spin of the atomic ground state. $\mathrm{N}$, having a ${ }^{4} \mathrm{~S}$ ground state, has therefore $\mathrm{g}_{\mathrm{A}}=(1)(4)=4$; oxygen, with a ${ }^{3} \mathrm{P}$ state, has a weight $g_{A}=(3)(3)=9$. $G_{M}$ consists of three factors referring to the electronic state $\left(g_{M}\right)$ of the molecule, the vibration $\left(g_{V}\right)$ and the rotation $\left(g_{r}\right)$ respectively,

$$
G_{M}=g_{M} g_{V} g_{r}
$$


$g_{M}$ can be calculated from spin $S$ and orbital momentum $\Lambda$, viz.

$$
\begin{array}{ll}
\mathrm{g}_{\mathrm{M}}=2 S+1 & \text { for } \Sigma \text { states }(\Lambda=0) \\
2(2 S+1) & \text { for all other states }(\Lambda \neq 0)
\end{array}
$$

The ground state of $\mathbb{N}_{2}$ is a ${ }^{1} \boldsymbol{\Sigma}$ state $\left(\varepsilon_{M}=1\right)$, that of $O_{2}$ a ${ }^{3} \Sigma$ state $\left(g_{\mathrm{M}}=3\right)$. $\mathrm{g}_{\mathrm{r}}$ is always given with oufficlent approximation by classical kinetic theory

$$
g_{r}=\frac{k T}{\mathrm{hB}_{r}}
$$

where $B_{r}$ is defined by the fact that the rotation levels of the molecule are $B_{r} j(j+1)$ if $j$ is the rotational quantum number; $B_{r}=1.44 \mathrm{~cm}^{-1}$ for $O_{2}$ and $2.00 \mathrm{~cm}^{-1}$ for $\mathbb{N}_{2}$. If $B_{r}$ is measured in $\mathrm{cm}^{-1}$ and $T$ in degrees Kelvin, $g_{r}=T / 1.438 B_{r}$. The vibrational part, $g_{v}$, is given by

$$
B_{\mathrm{v}}=\frac{1}{1-e^{-z}}
$$

with $z$ defined as in (1.5a). Ordinarily, dissociation is only important at high temperatures; then $g_{\nabla}$ is sufficlently nearly

$$
\mathrm{g}_{\mathrm{v}}=\mathrm{kT} / \mathrm{h} \nu
$$

If there are several modes of vibration, there is one factor of the type (1.13) for each mode.

Wher $\mathrm{K}$ has been calculated, and the total pressure $\mathrm{p}$ is know, $\alpha$ can be calculated immediately from (1.7), viz.

$$
\alpha=\sqrt{\frac{K}{K+4 p}}
$$


In the theory of shock waves, the density $\rho$ on the high pressure side can be more readily estimated than the pressure (par.3). Then, inserting (1.2) in (1.7), we heve

$$
\text { 4RT } \rho \alpha^{2}(1+\alpha)=K\left(1-\alpha^{2}\right)
$$

which gives

$$
\alpha=-1 / 2 K^{\prime}+\sqrt{K^{\prime}+1 / 4 K^{\prime 2}}
$$

with

$$
K^{\prime}=\frac{K}{4 R T \rho}
$$

If $\rho_{0}$ is the density of the gas at temperature $T_{0}$ and untt pressure, we have

$$
K^{\prime}=\frac{K}{4} \frac{T_{0}}{T} \frac{p_{0}}{\rho}
$$

The dissociation $\alpha$ depends strongly on the temperature (increasing with increasing $T$ ) and slightly on the pressure or density (decreasing with increasing $p$ or $p)$. The equation of state for a dissociated gas has already been given in (1.2). The energy content is given by

$$
\beta=\frac{1-\alpha}{1+\alpha} \quad \beta_{M}+\frac{\alpha}{1+\alpha} \quad\left(\frac{D}{R_{O} T}+2 \beta_{A}\right)
$$

where $\beta_{M}$ and $\beta_{A}$ are the coefficients of energy content for the molecular and the atomic gas at the given temperature. Disregarding the term $D / R_{0} T,(1.16)$ is simply the weighted average of $\mathrm{B}_{\mathrm{M}}$ and $\mathrm{B}_{\mathrm{A}}$, the weights being given by the partial pressures. The dissociation itself contributes an energy $\alpha D$ per mol of the gas; to obtain $\beta$, the energy per mol must be divided by $\mathrm{Mp} / \rho=(1+\alpha) R_{0} T$ ( $M$ the molecular weight). $\beta_{M}$ can be calculated by adding the contributions 1 to 4 discussed above. $\beta_{A}$ is essentially due to translational energy only, and has therefore the value 5/2. (The energy of electronic excitation of the atoms is seldom important below $5,000^{\circ}$ and has therefore been neglected.) 
At extremely high temperatures (above 5,000') the ionization of atoms and molecules must be considered. This can be done using similar calculations as for dissociation.

\section{Tables}

Table II gives the energy constant $\beta$ of nitrogen and oxygen at temperatures from 300 to $5000^{\circ}$ Kelvin. The energy constant from $300^{\circ}$ down to the IIquefaction temperature remains almost unchanged. The data for nitrogen were taken from W. F. Giauque and J. O. Clayton, Journ. Am. Chem. Soc. 55, 4875 (1933), those for oxygen from H. J. Johnston and M. K. Walker, 1bld. 55, 172 (1933). Both sets of data were calculated by the respective authors taking into account all corrections such as anharmonlcity of the vibrations, interaction between rotation and vibration, and electronic excitation. The figures given in our table for nitrogen are less accurate because Glauque and Clayton give only the free energy from which the energy content had to be obtained by numerical differentiation, involving considerable inaccuracy. A graphical method was used to smooth out the results of the numerical differentiation. The energy content of $\mathrm{O}_{2}$ could be read directly from the tables of Johnston and Walker as the difference between $T$ times the entropy, and the free energy.

The dissociation was calculated for oxygen and nitrogen in air of a density equal to 8 times the density at $300^{\circ} \mathrm{K}$ and one atmosphere pressure. These conditions were chosen because in a shock wave in which the temperature is raised to $3000-5000^{\circ}$, the density is increased about 8 fold (cf. 3, Table VIII). Obviously, the figures for oxygen would also be valid for pure oxygen of a density of $(8)(0.210)=1.680$ times that at $300^{\circ}$ and one atmosphere, and the dissoclation of nitrogen would be the same for pure nitrogen of a density of $(8)\left(0.780_{5}\right)=6.244$ times the density of nitrogen at $300^{\circ} \mathrm{K}$ and one atmosphere. 
Table II. Energy Content and Dissoclation of Nitrogen and Oxygen

Nitrogen

\begin{tabular}{|c|c|c|c|c|c|c|c|c|}
\hline $\mathbf{T}$ & $\beta_{M}$ & $\mathbf{K}$ & $\alpha$ & $\beta$ & $\beta_{M}$ & $\mathbf{K}$ & $\alpha$ & $\beta$ \\
\hline 300 & 3.493 & & & 3.493 & 3.493 & & & 3.493 \\
\hline 400 & 3.499 & & & 3.499 & 3.520 & & & 3.520 \\
\hline 500 & 3.508 & & & 3.508 & 3.548 & & & 3.548 \\
\hline 600 & 3.521 & & & 3.521 & 3.590 & & & 3.590 \\
\hline 700 & 3.541 & & & 3.541 & 3.636 & & & 3.636 \\
\hline 800 & 3.564 & & & 3.564 & 3.684 & & & 3.684 \\
\hline 900 & 3.594 & & & 3.594 & 3.731 & & & 3.731 \\
\hline 1000 & 3.625 & & & 3.625 & 3.774 & & & 3.774 \\
\hline 1250 & 3.702 & & & 3.702 & 3.871 & & & 3.871 \\
\hline 1500 & 3.780 & & & 3.780 & 3.950 & & & 3.950 \\
\hline 1750 & 3.845 & & & 3.845 & 4.015 & & & 4.015 \\
\hline 2000 & 3.900 & $4.0 \cdot 10^{-14}$ & $1.55 \cdot 10^{-8}$ & 3.900 & 4.068 & $5.15 \cdot 10^{-7}$ & $1.07 \cdot 10^{-4}$ & 4.071 \\
\hline 2500 & 3.992 & $4.9 \cdot 10^{-10}$ & $1.53 \cdot 10^{-6}$ & 3.992 & 4.157 & $2.36 \cdot 10^{-4}$ & $2.05 \cdot 10^{-3}$ & 4.199 \\
\hline 3000 & 4.062 & $2.61 \cdot 10^{-7}$ & $3.23 \cdot 10^{-5}$ & 4.063 & 4.223 & 0.0142 & 0.0144 & 4.454 \\
\hline 3500 & 4.127 & $2.28 \cdot 10^{-5}$ & $2.86 \cdot 10^{-4}$ & 4.134 & 4.278 & 0.268 & 0.0568 & 4.982 \\
\hline 4000 & 4.177 & $6.95 \cdot 10^{-4}$ & $1.44 \cdot 10^{-3}$ & 4.205 & 4.327 & 2.45 & $0.152_{3}$ & 5.79 \\
\hline 500 & 4.218 & $9.85 \cdot 10^{-3}$ & $5.12 \cdot 10^{-3}$ & 4.304 & 4.374 & 13.8 & 0.308 & 6.58 \\
\hline 5000 & 4.253 & $8.22 \cdot 10^{-2}$ & $1.39 \cdot 10^{-2}$ & 4.457 & 4.412 & 55.4 & 0.500 & 7.07 \\
\hline
\end{tabular}

\section{Oxygen}


It is seen that for oxygen the increase of the energy content, both due to vibrations and to dissociation, begins at much lower temperatures than for nitrogen. At $5000^{\circ}$, the values of $\beta$ differ by more than 50 per cent. This is due mainly to the smaller dissoclation energy and vibrational frequency of $\mathrm{O}_{2}$, and to a small extent also to the smaller concentration of $\mathrm{O}_{2}$ in air (cf. 1.15a).

Table III gives the necessary data for a1r. We have assumed a composition of

$$
\begin{aligned}
78.05 \text { per cent } & \text { N1trogen } \\
21.00 \text { per cent } & \text { Oxygen } \\
0.92 \text { per cent } & \text { Rare gases } \\
0.03 \text { per cent } & \mathrm{CO}_{2}
\end{aligned}
$$

all percentages being by volume, 1.e. by number of molecules. The energy content of the rare gases is $\beta=5 / 2$ because they are monatomic. The $\beta$ of $\mathrm{CO}_{2}$ was only guessed because of Its small concentration.

From the $\beta^{\prime} s$ and $\alpha^{\prime} s$ of the constituent gases, the dissociation and the energy content of a mixture are calculated as follows:

$$
\begin{array}{r}
\alpha=\sum_{k} c_{k} \alpha_{k} \\
\beta=\frac{\sum_{k} c_{k} \beta_{k}\left(1+\alpha_{k}\right)}{1+\alpha}
\end{array}
$$

where $c_{k}$ is the concentration (by volume) of the $k$ th component of the mixture $\left(\Sigma_{k} c_{k}=1\right), \alpha_{k}$ and $\beta_{k}$ its degree of dissociation and energy constant, respectively. $\alpha$ is mainly important for the calculation of $p / p, E q(1.2)$.

\section{Spec1f1c Heat}

The specific heat can be obtained by differentiating the energy content. As long as there is no dissociation, the specific heat per gram at constant pressure is 


$$
c_{p}=\frac{d}{d T}(R T \beta)=R\left(\beta+T \frac{d \beta}{d T}\right)
$$

The velocity of sound, again in the absence of dissociation, is given by

$$
a^{2}=\gamma R T
$$

where is the ratio of the specific heats at constant pressure and constant volume, viz.

$$
\gamma=\frac{c_{p}}{c_{v}}=\frac{c_{p}}{c_{p}-R}
$$

If $\beta$ is independent of temperature, we may write from (1.19) and (1.21):

$$
\beta=\frac{c_{p}}{R}=\frac{\gamma}{\gamma-1}
$$

as is commonly done in the theory of shock waves and other phenomena involving gases in rapid motion. While this is approximately justified for low temperatures, it is certainly not for air above $600^{\circ} \mathrm{K}$.

We have not included the specific heat in our Tables II and III because another numerical differentiation would have been necessary which would have made the results very inaccurate. Moreover, we belleved that there was at the moment no pressing need for a table of the specific heat and of the velocity of sound at very high temperatures but that the interest was centered around the shock waves. If there is dissociation, (1.19) is no longer correct because the energy is RT $\beta(1+\alpha)$ and $\alpha$ as well as $\beta$ changes with temperature. Moreover, the derivative with respect to $T$ must now be calculated at constant pressure. Furthermore, the difference between $c_{p}$ and $c_{v}$ is no longer $R$ so that (1.2I) is no longer valid. 
Table III. Energy Content and Dissociation of Air

\begin{tabular}{|c|c|c|c|c|c|c|c|}
\hline $\mathbf{T}$ & $\mathbb{N}$ & $\begin{array}{c}c_{k} \beta_{k} \\
0\end{array}$ & $\begin{array}{c}\left(1+\alpha_{k}\right) \\
\text { Rare } \\
\text { gases }\end{array}$ & $\mathrm{CO}_{2}$ & $\beta(1+\alpha)$ & $\alpha$ & $\beta$ \\
\hline 300 & 2.726 & 0.733 & 0.023 & 0.001 & 3.483 & & 3.483 \\
\hline 400 & 2.731 & 0.739 & $"$ & $"$ & 3.494 & & 3.494 \\
\hline 500 & 2.738 & 0.745 & $"$ & $"$ & 3.507 & & 3.507 \\
\hline 600 & $2.748_{5}$ & 0.754 & $"$ & " & 3.527 & & 3.527 \\
\hline 700 & 2.764 & 0.764 & " & $0.00 I_{5}$ & 3.552 & & 3.552 \\
\hline 800 & $2.781_{5}$ & 0.774 & " & " & 3.580 & & 3.580 \\
\hline 900 & 2.805 & $0.783_{5}$ & $"$ & $"$ & 3.613 & & 3.613 \\
\hline 1000 & $2.829_{5}$ & $0.792_{5}$ & $"$ & $"$ & 3.647 & & 3.647 \\
\hline 1250 & $2.889_{5}$ & 0.813 & " & 0.002 & 3.727 & & 3.727 \\
\hline 1500 & $2.950_{5}$ & $0.829_{5}$ & " & $"$ & 3.805 & & 3.805 \\
\hline 1750 & 3.001 & 0.843 & $"$ & $"$ & 3.869 & & 3.869 \\
\hline 2000 & 3.044 & 0.855 & $"$ & $"$ & 3.924 & 0.0000 & 3.924 \\
\hline 2500 & 3.115 & $0.883_{5}$ & " & $0.002_{5}$ & 4.024 & 0.0004 & 4.023 \\
\hline 3000 & 3.171 & 0.949 & $n$ & $"$ & 4.145 & 0.0030 & 4.133 \\
\hline 3500 & 3.2275 & 1.106 & $"$ & $"$ & 4.359 & 0.0121 & 4.307 \\
\hline 4000 & 3.287 & 1.400 & $"$ & 0.003 & 4.713 & 0.0331 & 4.562 \\
\hline 4500 & $3.376_{5}$ & 1.808 & " & $0.003_{5}$ & 5.211 & 0.0687 & 4.875 \\
\hline 5000 & 3.527 & 2.226 & " & 0.004 & 5.780 & 0.1058 & 5.227 \\
\hline
\end{tabular}


Finally, (1.20) ceases to be correct and is replaced by

$$
a^{2}=\frac{c_{p}}{c_{v}}\left(1+\alpha+\left[\frac{\partial \alpha}{\partial \log p}\right]_{T} \text { RT }\right)
$$

2. The Approach of Equilibrium between Various Degrees of Freedom of the Molecules.

Suppose the energy content of a mass of gas is suddenly changed, as it

is when the gas passes through a shock wave. Then it will take some time until the various degrees of freedom adapt themselves to the new conditions, and this "time" of relaxation" will be different for the different degrees of freedom.

\section{A. Translation and Rotation.}

The equilibrium will be attained most rapidly by the translation. For this degree of freedom, one collision is in general sufficient to come close to equilibrium. In order to have conditions similar to those in a shock wave we may consider a gas of a certain temperature $T_{2}$, into which streams a more dilute gas of a lower temperature $T_{I}$. Then the molecules of the cooler gas will (on the avo erage) become accelerated as soon as they make their first collision with those of the hotter gas. The average kinetic energy of a molecule of a cool gas will increase from $3 / 2 \mathrm{kT}_{1}$ in one collision to something of the $\operatorname{order}\left(3 / 2 \mathrm{k} / 1 / 2\left(\mathrm{~T}_{1}+\mathrm{T}_{2}\right)\right.$. A shock wave can obviously never be quite discontinuous but the transition from temperature $T_{1}$ to $T_{2}$ takes place over a distance of at least one gaskinetic mean free path $\lambda_{t}$ (t for translation). For ordinary gases at room temperature and atmospheric pressure, $\lambda_{t}$ is of the order of $10^{-5} \mathrm{~cm}$; it is in first approximation independent of the temperature and inversely proportional to the density; therefore, even a very violent shock wave in which the density increases by a factor 6 to 10 (cf.par, 3 Table VIII), wust have an extension of at least about $10^{-6} \mathrm{~cm}$. The classical theory of the physical structure of shock waves as given by Becker (Zeits. f. Phys. 8, 321, 1922) gives extremely small extensions which become of the order of $10^{-7} \mathrm{~cm}$ for very violent waves. The theory of Becker which takes Into account the heat conduction but neglects nolecular effects can therefore 
not be correct, at least not for violent shock waves. Becker, himself, pointed out that the problem requires a treatment based on the kinetic theory of gases. In practice a spatial extension of the shock wave of the order of one mean free path is, of course, of no importance at all, even at rather low inftial pressures. The molecular rotation may approach equilibrium as rapidiy as the translation. This would be expected for strongly elongated molecules such as $\mathrm{CO}_{2}$. If the effective boundary of the molecule is nearly spherical (e.g. $\mathrm{N}_{2}$ or $\mathrm{s}_{2}$ ) the excitation of molecular rotations may be estimated to take roughly 10 to 100 collisions. To show that the rotation approeches equilibrium so quickly, we use the results of Landau and Teller, Physik. Zeits. d. Sowjetunion 10, 34(1936). These authors have found that the effectiveness of collisions on a certain degree of freedom is determined by the ratio

$$
x=\sigma_{c} / T_{0}
$$

where $\boldsymbol{T}_{c}$ is the effective duration of the collision and $\boldsymbol{T}_{\mathrm{o}}$ the natural period of the degree of freedom concerned. If $x$ is of order unity or smaller, one or a few collisions will be sufficient to establish equilibrium whereas a large number of collisions is required if $x \gg I$ (cf. 2.5$)$.

$r_{c}$ in $(2.1)$ may generally be written

$$
\tau_{\mathrm{c}}=s / v
$$

where $v$ is the relative velocity of the two colliding molecules and $s$ the range of the intermolecular forces, 1.e. the distance over vich the molecules interact strongly. We may expect s to be of the order of one helf to one Bohr radius, 1.0: 2.5 to $5: 10^{-9} \mathrm{~cm}$, a range of values which seems confirmed by some experimental results on the approach of vibrational equilibrium (cf. Table IV). In the case of rotation $\boldsymbol{T}_{0}$ may be taken as the time required for one revolution, or rather this. time divided by $2 \pi(c F .2 .4)$ so that $r_{0}=r / V_{I}$ where $r$ is the radius of the molo ecule (distance of an atom from the center of gravity) and $v_{r}$ is the velocity of 
the atoms in their revolution around the center of gravity。 Now $v_{r}$ is of the same order as $v$, the velocity of molecular translation (equipartition of energy:) and $r$ is of the same order as $s$. Therefore $X$ is of order unity for rotation, and equilibrium between rotations and translation will be attained in a few collisions. We shall find in the following that all other: degrees of freedom behave quite differently in that many collisions are necessary to establish equilibrium. Therefore 'it will be convenient to group together translation and rotation on one side, and all other degrees of freedom on the other. The latter we shall call the "Inert" degrees of freedom while translation and rotation will be denoted as the "active" degrees of freedom. For all practical purposes we may say that the energy content of the active degrees of freedom can change almost discontinuously, because a distance of a few mean free paths may be considered negligible. We can then define the temperature of a moving gas at each point by the energy content of the active degrees of freedom, which is

$$
E_{a}+p / \rho=\beta_{a} p / \rho=\beta_{a} R T
$$

(the last relation being only valid if there is no dissociation). In (2.3) $\beta_{a}$ is independent of the temperature and equal to $5 / 2$ for monatomic, $7 / 2$ for diatomic gases (cf. par. I). The energy content of the inert degrees of freedom, $E_{1}$, on the other hand, cannst change abruptly and is therefore not always in equilibrium with that of the active ones; in other words, $E_{1}$ is not necessarily related to the local. temperature $T$ in the way discussed in par. 1 .

\section{B. Vibrations}

Theory

The most important inert degree of freedom are the vibrations. For these, we set in (2.1)

$$
T_{0}=1 / 2 \pi \nu
$$


where $V$ is the natural frequency of the molecular vibration. (In all formulae Iike (2.1) $2 \pi \nu$ rather than $\nu$ should bo used as representing the frequency because the frequency is generally introduced into physical formulee by taking the time derivative of experselons like sin $2 \pi v t$. If we took $\nu$ instead of $2 \pi \nu$ in $(2.4)$, the only change would be that the values of 8 deduced from experfmental data would be multiplied by $2 x$ ). Since the frequencies of molecular vibrations are rather high, It is plausible that $\chi$ is rather large; this will be shown by direct calo culation and by discusilon of experimental results below. For the cese $x \gg 1$, Landau and Teller give the formula

$$
p_{10}=\operatorname{Ce}^{-\infty} x
$$

Here $p_{10}$ is the probability that a molecule in the first excited state of vibration is de-excited by a collision with another molecule. C is a geometrical factor which gives the probebility that the collision of the two molecules will take place in a direction suitable for excitation or de-excitation of the vibration. There are no experimental data oufficiently accurate to deduce $C, 80$ that qualitative arguments must be used for its determination. Obviously, $C$ mat be less than unity, and probably it will lie between $1 / 3$ and $1 / 30$ in most cases, its value being higher for diatomic and lover for polyatomic molecules beceuse it is less likely that a como plicated molecule is hit at the right plece to induce a given mode of vibration. In our computations below, we shell use arbitrarily $\mathrm{Cal} / 10$.

Presumably, a better approximation could be obtained by introducing in (2.5) another factor, viz. a certain porrer of $\mathcal{X}$. Arguments can be given for a factor $x^{\text {t2 }}$. Horrever, these arguments are too uncertain to justify at present the inclusion of such fino pointo.

The most important fector in $(2.3)$ is the exponential $\theta^{*} \mathrm{~K}$ where (cf. 2.1, 2.2, 2.4)

$$
x=2 \times 28 / v
$$


Let us investigate $\chi$ for the case when the kinetic energy of the relative motion of the colliding molecules is just $\mathrm{kT}, 1 . e$.

$$
1 / 2 M v^{2}=k T
$$

where $M$ is the reduced mass of the two molecules; if they are equal, $M$ is one half the mass of one molecule. The value of $\boldsymbol{X}$ for the velocity determined by (2.7) is

$$
X_{1}=2 \pi \nu s \quad \sqrt{M / 2 k T}
$$

This can be re-written as follows:

$$
x_{I}=\sqrt{h / 2 k T} \cdot s \sqrt{\frac{4 \pi^{2} M \nu}{h}}
$$

For the collision of two equal diatomic molecules, each consisting of two equal atoms, $M$ is the mass of one atom; then the reciprocal of the last factor is (2.9) viz.

$$
b=\sqrt{h / 4 \pi^{2} M \nu}
$$

represents the amplitude of the molecular vibration in the lowest quantum state. Generally, the $b$ defined in (2.10) has the value

$$
b=\frac{8.2 \cdot 10^{-8}}{\sqrt{\mu \nu}} \mathrm{cm}
$$

where $\boldsymbol{\mu}$ is the molecular welght of the molecule (assuming collision between equel molecules) and $\nu$ the vibrational frequency in $\mathrm{cm}^{-1}$. For $\mathrm{N}_{2}, \nu=2345$ and $\mu=28$ 80 that $b=3 \cdot 1 \cdot 10^{-10} \mathrm{~cm}$. Thus we see that $b 18$ very small compared with the range $s$ of the forces. The factor $\sqrt{h y / 2 k T}$ is (2.9) is also in general greater than unity, so that $X_{I}$ is indeed very large compared with unity as we expected above. Therefore (cf. 2.5) the probability of transfer of energy between vibration and 
trenslation is very small; the vibration is an inert degree of freedom. It is seen from the derivation that the reason for this result is not so much that the energy of one vibrational quantum $h \nu$ is large compared with the average energy of translation, $k T$, but rather that the amplitude of the vibrations, $b$, is very small compared with the range $s$ of the intermolecular forces. This in turn is due to the large elastic forces which govern the elastic vibrations.

The quantity $\boldsymbol{X}(2.6)$ will be reduced, and therefore the probability of energy transfer $p_{10}(2.5)$ considerably increased, if we take higher velocities $v$. Therefore a given molecule will lose and gain vibrational energy mostly at the times when its, kinetic energy of translation is high compared with $\mathrm{kT}$, i.e. when it is in the tail of the Maxwell distribution. If we average over all the molecules in the gas, the probability of energy transfer per collision becomes

$$
P_{10}=2 / \sqrt{\pi} \int e^{-x} \sqrt{x} d x C_{e}^{-x}
$$

where

$$
x=\frac{M v^{2}}{2 k T}
$$

(2.12) represents the probability (2.5), averaged over the Maxwell distribution of the relative velocities of the two colliding molecules.* With (2.6) for $\boldsymbol{x}$, the integration of (2.12) can be carried out by the saddle point methods, the integrand having a steep maximum near

* It might be preferable to take into account the different collision probability of fast and slow molecules, 1.e. to replace (2.12) by

$$
P_{10}=\int e^{-x} x d x c e^{-x}
$$

which would give instead of (2.13)

$$
P_{10}=2 / 9 \sqrt{\pi} \sigma^{3 / 2} e^{-\sigma}
$$

The temperature dependence of $P_{10}$ would not be changed very much by this correction because the difference between $(b)$ and (2.13) would be largely compensated by $a$ different value of $s$ deduced from the experiments. 


$$
x_{0}=1 / 2(2 \pi s \nu) 2 / 3(M / k T) 1 / 3
$$

The integration gives

where

$$
\begin{gathered}
p_{10}=C^{\prime} \sigma e^{-\sigma} \\
\sigma=3 x_{0}=3 / 2(\mathrm{~s} / \mathrm{b})^{2 / 3}(\mathrm{~h} \nu / \mathrm{kT}) 1 / 3 \\
\mathrm{C}^{\prime}=\frac{4}{(3)} 3 / 2 \mathrm{C}
\end{gathered}
$$

( $b$ as defined in $(2.10,2.100)$, s the range of the molecular forces, $\nu$ the frequency of vibration). (Fqs. $(2.13,14$ ) are similar to the equations determining the rate of nuclear roactions in stars).

NumericallJ, (2.14) may be written

$$
\sigma=0.090(\nu \mathrm{s})^{2 / 3}(\boldsymbol{\mu} / \mathrm{T}) 1 / 3
$$

where $\nu$ is measured in $\mathrm{cm}^{-1}$, $s$ in units of $10^{-9} \mathrm{~cm}_{3} \mathrm{~T}$ in degrees Kelvin, and $\mu$ is the molecular weight per molecule (or twice the reduced molecular weightg $2 \mu_{\mathrm{A}} \mu_{\mathrm{B}} /\left(\mu_{\mathrm{A}}+\mu_{\mathrm{B}}\right)$, if tro unequal nolecules collide). We shall now try to get a more quantitative estimate of $s$. Experiments are avaliable (cr. below), among other gaves, for pure $\mathrm{CO}_{2}$ and for the action of $\mathrm{N}_{2}$ on the vibretions of $\mathrm{O}_{2}$. For $\mathrm{CO}_{2}$ Fricke (Journ. of the Acoust. Soc。 of Am. 12, 245(1940)) find that $z=86,000$ collisions are necessary for de-excitation of the first excited vibrations state, so that

$$
P_{10}=\frac{1}{2}=\frac{1}{86,000}=1.150 .10^{-5}
$$

Taking $C^{*}=1 / 10,(2.13)$ gives $\sigma=11.5$. Joing this nuber in $(2.15)$ with $\nu$ $=667 \mathrm{~cm}^{-1}$, we rind,

$$
s=5.6 \cdot 10^{-9} \mathrm{~cm} \cdot\left(\mathrm{CO}_{2}\right)
$$


For the de-excitation of the vibrations of $\mathrm{O}_{2}$ by collisions with $\mathbb{N}_{2}$, Kneser and Knudsen find $P_{10}=10^{-5}$. Inserting this figure in $(2.13,15)$ and taking $V=1570$ $\mathrm{cm}^{-1}$, we abtain

$$
\mathrm{S}=3 \cdot 10^{-9} \mathrm{~cm}\left(\mathrm{O}_{2} / \mathrm{N}_{2}\right) .
$$

Both the results (2.16a) and $(2.16 \mathrm{~b})$ are of the order of the Bohr radius as we assumed above. Differences between the values of $\mathbf{s}$ for different molecules are, of course, to be expected. We can therefore not predict the value of $\mathbf{s}$ for a pair of molecules for which it has not been measured experimentally. This is very unfortunate because $P_{10}$, or

$$
z=1 / P_{10}
$$

is very sensitive to $\mathrm{s}$. This can be seen directly from $(2.13,14)$ or from Table IV In which we have calculated $\mathrm{Z}$ from $(2.13,14)$ for $\mathrm{O}_{2}$ and $\mathrm{N}_{2}$, in each case for two different values of s. A more detailed discussion of Table IV will be given in par。2D.

\section{Influence of foreign gases, experimental difficulties.}

Collisions between two different molecules are often more effective in transferring energy to and from the vibrations than collisions between like molecules. This is the case especially (1) if the two colliding molecules have a chemical affinity and (2) if one molecule is very light so that its velocity is great. In case (2) which is realized for $\mathrm{H}_{2}, \mathrm{He}$, etc., $\mu$ in (2.15) is very small. In case (1) the interaction between the two molecules is much more intense than usually which may perhaps result in more sudden changes of interaction and correspondingly shorter effective range, or even in a complete fallure of the Landau-Teller theory when the two molecules penetrate so deeply into each other that they can be sald to form a temporary compound. In the latter case, the temperature dependence may be quite different from that indicated in (2.13).

$$
\therefore: 31
$$


Table IV. Theoretical Temperature Dependence of the Number of Collision Necessary for

De-excitation of the First Vibrational State.

$$
\text { Temperature }\left({ }^{\circ} \mathrm{K}\right) \quad 300 \quad 500 \quad 700 \quad 1000 \quad 1500 \quad 2000 \quad 3000 \quad 5000
$$

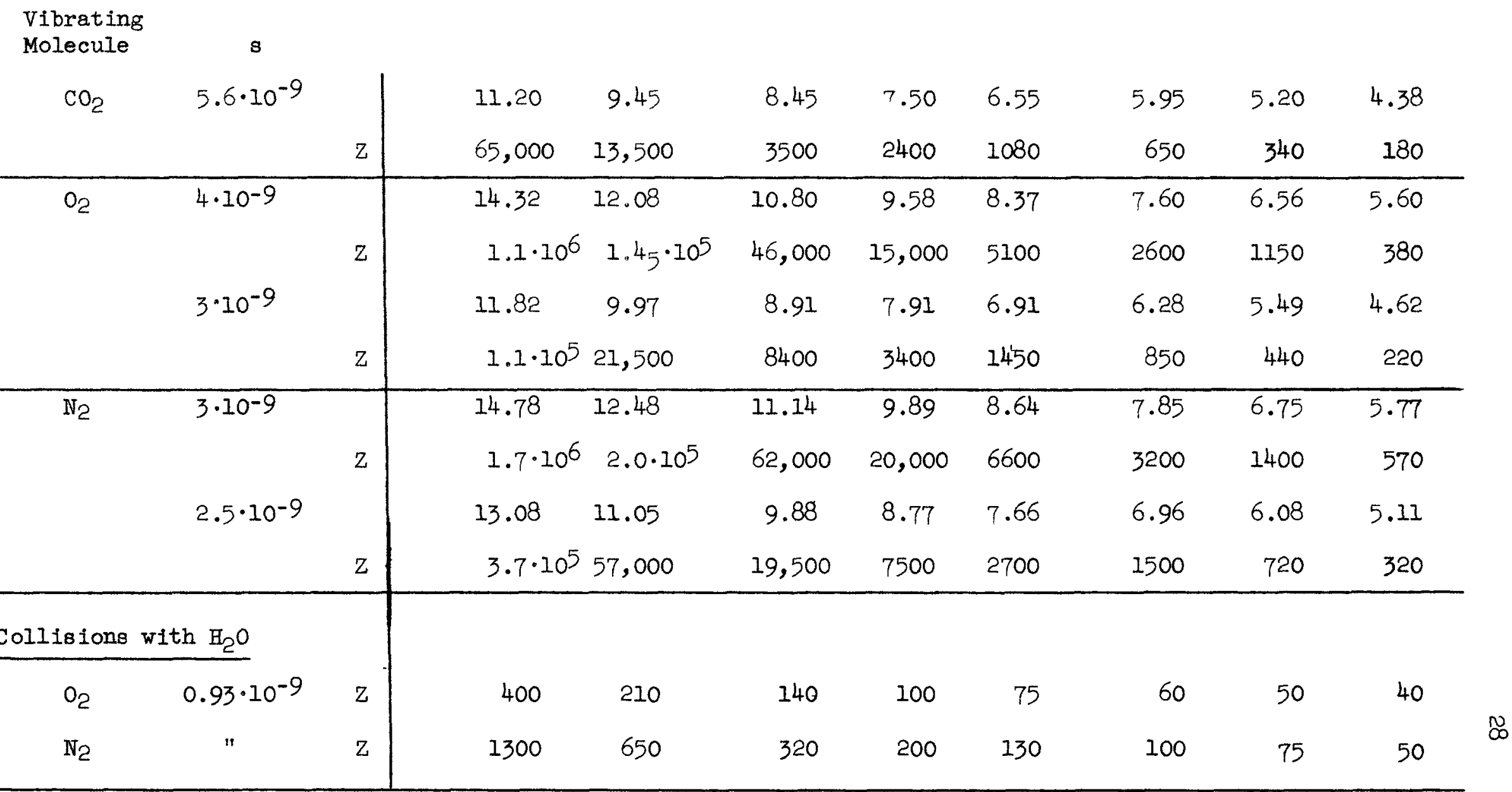

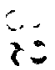


As will be seen from Table $\nabla$, the foreign gases investigated are 3 to 4000 times more effective in de-exciting the vibration of $\mathrm{O}_{2}$ than $\mathrm{O}_{2}$ itself, the effect being greatest for complicated molecules such as $\mathrm{C}_{2} \mathrm{H}_{2} \mathrm{OH}$ which has a chemical affinity to $\mathrm{O}_{2}$, and for water, which can probably form a temporary compound with $\mathrm{O}_{2}$. The effect of $\mathrm{N}_{2}$ on $\mathrm{O}_{2}$ is almost as small as that of $\mathrm{O}_{2}$ itself because there is not much chemical interaction; the colligions between $\mathrm{O}_{2}$ and $\mathrm{N}_{2}$ will be discussed in more detail in par. 2D.

The large effect of water vapor on the variation of oxygen makes it extremely difficult to measure the small effect of collisions between $\mathrm{O}_{2}$ molecules themselves. Only a lower limit for $\mathrm{z}$ was therefore obtained in the experiments on "pure" $\mathrm{O}_{2}$,

For the problem of shock waves we must conclude that the establishing of vibrational equilibrlum will depend sensitively on the humidity of the air. On hot hamid days, the water vapor content of the air may easily reach 3 per cent (23 mm vapor pressure) so that only $400 / 0.03=13,000$ collisions would be necessary to establish vibrational equilibrium for the oxygen. On the other hand, for completely dry air the necessary number of collisions is about $10^{5}$ because then only the collisions with nitrogen will be important. A more accurate discussion of the humidity effect will be given in Table VI.

Excitation and De-excitation. Instead of the probability $P_{10}$ of deexcltation per collision, it is convenient to introduce the probability $k_{10}$ of de-excitation per second which is given by

$$
\mathrm{K}_{10}=\mathrm{P}_{10} \cdot \mathrm{N}=\mathrm{N} / \mathrm{Z}
$$

where

$$
N=N q \quad v
$$

is the number of collisions per second, $N$ the number of molecules per $\mathrm{cm}^{3}, g$ the gas-kinetic collision cross section and $\bar{v}$ the arerage relative velocity of two 
colliding molecules, viz. $\sqrt{\frac{8}{\pi} \frac{\mathrm{kT}}{\mathrm{M}}}$. The quantity $\mathrm{k}_{10}$ has two edvantages compared with $\mathrm{P}_{10}$, viz. (I) that it is more directly related to the extension of the shock wave and (2) that it is directly connected with observational data (cf. 2.22, 26) whereas the connection of $\mathrm{P}$ with these data involves the somewhat uncertain cross section $q$.

The probability of excitation, $k_{01}$, is connected with the probability of de-excitation, $k_{10}$, by the statistical principle of detailed balancing according to which

$$
k_{01}=k_{10} e^{-\frac{h \nu}{k T}}
$$

The time rate of change of the number $y_{0}$ of molecules without vibration is then

$$
\frac{d y_{0}}{d t}=k_{10} y_{1}-k_{0 I} y_{0}
$$

where $y_{1}$ is the number of molecules in the first excited vibrational state. Similar equations hold for the other $y_{n}$ where, according to quantum theory, $k_{n}, n-1=$ $n k_{10}$ and $k_{n-1, n}=k_{n, n-1} e-h \nu / k T$. By adding the equations for the various $y_{n}$, an equation for the total energy of vibration,

$$
E_{v}=h \nu \sum_{n=0}^{\infty} n y_{n}
$$

can be obtained, namely

$$
\frac{d E_{V}}{d t}=k_{10} \quad\left(I-e^{-n \nu / k T}\right)\left(E^{\prime}{ }_{V}-E_{V}\right)
$$

Here $\mathrm{E}^{\prime} \mathrm{v}$ is the vibrational energy in thermal equilibrium, viz.

$$
E_{v}^{\prime}=\frac{h \nu}{e^{h \nu / k T}-1}
$$


Eq. (2.20) is valid no matter how large the deviation from thermal equilibrium. The factor $1-e-h \quad / k T$ takes account of the fact that there occur transitions away from equilibrium as well as towards equilibrium. The solution of (2.20) is

$$
E_{V}^{\prime}-E_{V}=A e^{-\omega_{0} t}
$$

where $\omega_{0}$ is the reciprocal of the time of relaxation and is given by (cf. 2.20).

$$
\omega_{0}=k_{10}\left(1-e^{-h \nu / k T}\right)
$$

It has been assumed in (2.21) that $T$ remains constant. This not strictly correct in shock waves (cf. par.3,4) where $\omega_{0}$, being a function of $T$, will change with time so that the integral of (2.20) cannot be given in closed form.

$$
\text { In shock waves, we are interested in the spatial variation of } \mathrm{E}_{\mathrm{v}^{*}} \text { If }
$$

the gas flows with a velocity $v$, we may write

$$
\frac{d E_{v}}{d x}=\frac{1}{v} \frac{d E v}{d t}=\frac{E_{v}^{\prime}-E_{v}}{\lambda_{v}}
$$

where

$$
\lambda_{\mathrm{v}}=v_{i} \omega_{0}
$$

defines the mean free path for vibration.

$$
\text { Evaluation of Experiments. Fne experimental determination of } \boldsymbol{\omega}_{0} \text { is }
$$
based upon the absorption and dispersion of sound in gases. The theory of this phenomenon has been given by H. O. Kneser, Ann. d. Phys. 16, 337 (1933) and Journ. Acoust. Soc. Amer. 2, 122 (1933), and others. The absorption coefficientaper wave length depends on the circular frequency $\omega_{\Lambda}$ of the sound wave approximately* as

* In order to obtain (2.25), $c_{p}-c_{p a}$ must be assumed to be small in comparison with $c_{p}$. Since $\mu$ is the absorption coefficient per wave length, the absorption coefficlent per centimeter will behave as

$$
\omega_{\max }^{2}+\omega^{2}
$$
and will therefore obtain its $\therefore 25$ maximum value for $\omega=\infty$. 


$$
\mu \approx \frac{\omega}{\omega_{\max }^{2}+\omega^{2}}
$$

where $\omega_{\max }$ is the frequency of maximum absorption per wave length. $\omega_{\max }$ can be determined experimentally, and $\omega_{0}$ may be deduced from it using the relation

$$
\frac{\omega_{0}}{\omega_{\max }}=\frac{c_{p a}\left(c_{p a}-R\right)}{c_{p}\left(c_{p}-R\right)} \text {. }
$$

Here $c_{p}$ is the ordinary specific heat at constant pressure and $c_{p e}$ the specific heat counting the "active" degrees of freedom only. In contrast to (2.25), (2.26) Is exact.

Experimental Results. Experiments were made by H. 0 . Kneser and V. 0 . Knudsen, Ann. d. Physik 2l, 682 (1935) on the vibrations of $0_{2}$, by Fricke and by Knudsen and Fricke, Journ. Acoust. Soc. Amer. 12, 245 and 255 (1940) on $\mathrm{CO}_{2}$ and a few other gases, and by Küchler, Zeits. f. phys. Chemie B 41 , 199 (1938), on the temperature dependence of the time of relaxation. The latter experiments were probably carried out with somewhat impure gases because küchler finds for $\mathrm{CO}_{2}$ at room temperature $\mathrm{Z}=50,000$ whereas Fricke gives 86,000 (Impurities reduce $\mathrm{z}$, cf. above and Table $\mathrm{V}$ ), and a similar discrepancy exlsts for $\mathrm{N}_{2} \mathrm{O}(7,500 \mathrm{\nabla s} .11,800)$. The experiments of Kneser and Knudsen were the first systematic ones carried out and were therefore less accurate than the later work of Knudsen and Fricke, but the results of Kneser and Knudsen are most important for us because they were done with oxygen. For $\mathbb{N}_{2}$ there are no experiments but only an estimate by $P . S . H$. Henry, Nature 129, 200 (1932) based on the fallure to detect the Influence of the vibrational specific heat on the velocity of sound in certain experiments. Henry estimates $\omega_{0} \approx 10^{4}$ sec $^{-1}$, i.e. $\mathrm{Z} \approx 10^{6}$ for $\mathrm{N}^{2}$ which seems not implausible.

Table V gives some of the experimental results. They bear out the features discussed above and expected theoretically, viz. 
ilable V. Experimental Results on the Excitation of Molecular Vibrations. Number of Collisions Required for De-excltation, $Z$, and Reciprocal Time of Relaxation, $\omega_{0}$ (in $\mathrm{sec}^{-1}$ ), for Various Molecules $C$ olliding. with other Molecules.

\begin{tabular}{|c|c|c|c|c|c|c|c|c|c|}
\hline Collision with & & $\mathrm{O}_{2}$ & $\mathrm{~N}_{2}$ & $\mathrm{H}_{2}$ & $\mathrm{CO}_{2}$ & $\mathrm{H}_{2} \mathrm{O}$ & $\mathrm{H}_{2} \mathrm{~S}$ & $\mathrm{CH}_{3} \mathrm{OH}$ & $\mathrm{C}_{2} \mathrm{H}_{5} \mathrm{OH}$ \\
\hline $\begin{array}{l}\text { Vibration of } \mathrm{O}_{2} \\
\text { (Knudaen and Kneser) }\end{array}$ & $\omega^{z}$ & $\begin{array}{l}=500,000 \\
=3 \cdot 10^{3}\end{array}$ & $\begin{array}{l}100,000 \\
5 \cdot 10^{4}\end{array}$ & $\begin{array}{l}20,000 \\
5 \cdot 10^{5}\end{array}$ & $\begin{array}{l}25,000 \\
1.7 \cdot 10^{5}\end{array}$ & $\begin{array}{c}400 \\
1.1 \cdot 10^{7}\end{array}$ & $\begin{array}{r}4,200 \\
1.3 \cdot 10^{6}\end{array}$ & -- & $\begin{array}{c}120 \\
6 \cdot 107\end{array}$ \\
\hline $\begin{array}{l}\text { Vibration of } \mathrm{CO}_{2} \\
\text { (Kneser and Fricke) }\end{array}$ & $\mathrm{z}$ & $-\cdots$ & $\cdots$ & $\begin{array}{r}215 \\
8.0^{*} 10^{7}\end{array}$ & $\begin{array}{l}86,000 \\
9.8 \cdot 10^{4}\end{array}$ & $\begin{array}{c}17 \\
1.02 \cdot 10^{9}\end{array}$ & $\begin{array}{l}1,200 \\
1.14 \cdot 10^{7}\end{array}$ & $\begin{array}{l}36 \\
3.1 \cdot 10^{8}\end{array}$ & $--\cdots--$ \\
\hline
\end{tabular}

The same quantities for some pure geses (Fricke)

\begin{tabular}{crrrrrr} 
Gas & \multicolumn{1}{c}{$\mathrm{O}_{2}$} & $\mathrm{CO}_{2}$ & $\mathrm{~N}_{2} \mathrm{O}$ & $\mathrm{COS}$ & $\mathrm{CS}_{2}$ & $\mathrm{SO}_{2}$ \\
\hline $\mathrm{z}$ & $>500,000$ & 86,000 & 11,800 & 9,600 & 8,700 & 1,900 \\
$\mathrm{o}^{\left(\text {in } 10^{5} \mathrm{sec}^{-1}\right)}$ & $<-.08$ & 0.98 & 6.9 & 11.5 & 14.3 & 55 \\
\hline
\end{tabular}

$\mathrm{Z}$ for $\mathrm{CO}_{2}$ at various temperatures (Küchler)

$\begin{array}{cccccc}\text { T(degrees Kelvin) } & 293 & 373 & 473 & 573 & 673 \\ \mathrm{Z} & 50,000 & 31,000 & 19,000 & 12,000 & 9,000\end{array}$

Ratio of $z_{293^{\circ}} / z_{673^{\circ}}$ for various gases (Küchler)
Colliding gases
$\mathrm{CO}_{2}-\mathrm{CO}_{2}$
$\mathrm{N}_{2} \mathrm{O}-\mathrm{N}_{2} \mathrm{O}$
$\mathrm{CO}_{2}-\mathrm{B}_{2}$
$\mathrm{CO}_{2}-\mathrm{H}_{2} \mathrm{O}$

$\frac{z_{293^{\circ}}}{i} z_{293} / z_{673}$

$\begin{array}{ll}50,000 & 7,500 \\ 5.6 & 3.6\end{array}$

300

105

1.0

0.4 
1. All the pure gases investigated have rather highz (small $\omega_{\circ}$, long relaxation times), the smallest being $\mathrm{SO}_{2}$ with $\mathrm{Z} \approx 2,000$, the largest $\mathrm{O}_{2}$ with $z>500,000$. The large value for $\mathrm{O}_{2}$ is probably due mostly to its high vibration frequency. The decrease of $\mathrm{z}$ from $\mathrm{CO}_{2}$ to $\mathrm{COS}$ to $\mathrm{CS}_{2}$ is also in the direction of decreasing $\boldsymbol{\nu}$; on the other hand, the small value for $\mathrm{SO}_{2}$ is presumably due to the greater chemical activity of that molecule.

2. The impurities investigated gave smaller $Z$ than pure gases. It cannot be decided at present whether this is due to the selection of gases used in the experiments, or to a general rule. Among diatomic molecules, $\mathrm{H}_{2}$ is most effective in de-exciting $\mathrm{O}_{2}$ and $\mathrm{CO}_{2}$; this is to be expected theoretically from Its small mass (large velocity). Triatomic gases are on the whole more effective than diatomic ones; this may be due to the fact that there will always be some "corner" of a triatomic molecule which has a chemical affinity or at least a strong interaction with a given molecule. Among the triatomic gases, there is again a decrease of $\mathrm{Z}$ with increasing chemical activity $\left(\mathrm{CO}_{2}\right.$ to $\mathrm{H}_{2} \mathrm{~S}$ and $\left.\mathrm{H}_{2} \mathrm{O}\right)$. Polyatomic molecules are even more effective than triatomic ones, for the same reason. By collisions with the same molecule, the vibrations of $\mathrm{CO}_{2}$ are in general more affected than those of $\mathrm{O}_{2}$, because of the smaller frequency of vibration.

3. The temperature dependence of $\mathrm{Z}$ for $\mathrm{CO}_{2}$ is about as expected ( $\mathrm{cf}$. Table IV). Genfrally, the decrease of $Z$ with increasing temperature is most pronounced if $Z$ is large, in agreement with theoretical expectation. Whether the increase of $\mathrm{Z}$ with the temperature as found by Küchler for collisions between $\mathrm{CO}_{2}$ and $\mathrm{H}_{2} \mathrm{O}$, is real cannot be decided at present; however, $\mathrm{Z}$ is very small in this instance so that the Landau-Teller theory can probably not be applied.

Several Degrees of Freedom. At first sight, it might be expected that each mode of vibration has its own relaxation time, this time being greater for the modes with higher frequency. Experiments show, however, that this is not the 
case because all the experimental curves show only one absorption maximum with the absorption coefficient falling off on both sides according to (2.25). This behavior can easily be understood if we remember that the resolution of the vibration into normal modes is only an approximation which is correct only for exactly harmonic forces. The anharmonicity will $\mathrm{mix}$ the varlous normal modes in each vibrational quantum state. An extreme case of mixing is known in $\mathrm{CO}_{2}$ where the second excited state ( $\nabla$ ibrational quantum number* $n_{1}=2$ ) of the first mode of vibration $\left(\nu_{1}=667 \mathrm{~cm}^{-1}\right)$ is degenerate with the first quantum state of the second mode $\left(V_{2}=1336 \mathrm{~cm}^{-1}\right)\left(n_{2}=1\right)$ (cf., e.g., Sponer, loc. cit.). The anharmontcity causes an "Interaction" of the two resonating quantum states with the result that a splitting occurs into two states of considerably different frequency (1286 and $1388 \mathrm{~cm}^{-1}$ ). The form of vibration in each of these states is a combination of modes 1 and 2 with about equal amplitudes.

In other molecules, the mixing of different modes is usually less strong but it must always exist to some extent. Let us assume, e.g., that there is a mode of vibration with a high frequency $\nu_{2}$ which is between 3 and 4 times the frequency $V_{1}$ of another mode. Then the first excited state of mode $2\left(n_{2}=1\right)$ will contain some admixture of the fourth state of mode $1\left(n_{1}=4\right)$. In this case, the excitation of the state $n_{2}=1$ will not take place by direct transfer of energy from the translation, but the translation will excite in successive collisions the states $n_{1}=1,2$ and 3 , and finally, in a fourth collision, the state $n_{2}=1$. This mechanism avolds large energy transfers in one collision which are very improbable according to the Landau-Teller theory (cf. 2.15). The transition from $n_{1}=3$ to $n_{2}=1$ is somewhat less probable than a collision in which $n_{1}$ is raised by one unit because the mixing between $n_{2}=1$ and $n_{1}=4$ is assumed small; on the other hand, it is more probable because the energy difference between $n_{2}=1$

* In order to avold confusion with the velocity $\nabla$, we denote the vibrational quantum number by $n$ rather than the customary $v$. 
and $n_{1}=3$ is smaller than $\mathcal{L}_{1}$. The $z$ for the transition $n_{1}=3$ to $n_{2}=1$ is therefore probably of the same order or smaller than for the excitation of the first node, and it can therefore be understood that only the successive excitation of quantum states of the lowest mode of vibration gives rise to an observable absorption of sound.

\section{Dissociation}

The dissociation differs from other degrees of freedom in that it becomes appreciable at temperatures at which kf is still very small compared with the dissoclation energy $D$ per molecule. We have mentioned in par. 1 that $\frac{D}{k T}=\frac{59,000}{T}$ for oxygen and 91,600/T for nitrogen. 'On the other hand, we have shown in Table II that the degree of dissociation is as much as 1.4 per cent for $\mathrm{O}_{2}$ at $3000^{\circ} \mathrm{K}$ and for $\mathrm{N}_{2}$ at $5000^{\circ} \mathrm{K}$. At these temperatures, $\mathrm{D} / \mathrm{kT}$ is about 20 in both cases, and the Boltzmann factor $e^{-\frac{D}{k T}} \approx e^{-20} \approx 10^{-9}$. The fact that an appreciable dissoclation is possible for such a small Boltzmann factor is due to the large a priori probability of the dissoclated states.

In order to produce dissoclation, two molecules must collide which have a relative kinetic energy at least equal to $D$. Such molecules are very rare because of the small Boltzmann factor $e^{-\frac{D}{k I I}}$. Dissociation will therefore take a considerable time at $3000-5000^{\circ} \mathrm{K}$ even if every collision between molecules of sufficient energy is effective.

The same conclusion can be reached by considering the inverse process, viz. recombination. In order that two atoms recombine into a molecule, there must be a triple collision between the two atoms and another molecule which takes up the excess energy and momentum. Triple collisions, however, are rare events especially if two of the colliding particles must be atoms which are relatively rere as long as the degree of dissociation remains low.

We must now examine the efficiency of collisions between two molecules of sufficlent relative velocity in causing dissociation of one of the molecules. 
If two molecules have relative kinetic energy equal to $D$, $1 . e$. several electronvolts, they w1ll penetrate very deeply into each other. In this case, we can no longer distinguish between fast motions (of the electrons) and slow motions (of the molecules as a whole), and we can therefore no longer conclude that the transfer of energy from the slow molecular translation to the fast electronic motion is improbable. It is very difficult to make any quant1tative estimates but we belleve that the efficiency of collisions between molecules of energy greater than $D$ will not be reduced by a factor of the type of (2.5) but will be determined mainly by a geometrical factor which may perhaps be somewhat smaller than for the exc1tation of vibrations. In numerical calculations, we shall assume an efficlency $C_{d}=1 / 100$ which may be wrong by a factor of 10 or more either way.

The probability that the relative kinetic energy of a pair of molecules 1s between $\mathrm{kTx}$ and $\mathrm{kT}(\mathrm{x}+\mathrm{dx})$ is given by the Maxwell distribution

$$
\frac{2}{\sqrt{\pi}} \sqrt{x} d x e^{-x}
$$

The velocity of the molecules in question is $\frac{\sqrt{\pi}}{2} \sqrt{x}$ times the average relative velocity $\bar{v}$ of two molecules. Therefore, the fraction of all molecular collisions for which the relative kinetic energy of the colliding molecules lies in the interval mentioned is approximately

$$
f(x) d x=e^{-x} x d x
$$

The fraction of collisions for which

$$
\mathrm{x}>\mathrm{x}_{0} \equiv \frac{\mathrm{D}}{\mathrm{kT}}
$$

is then

$$
F\left(x_{0}\right)=\int_{x_{0}}^{\infty} f(x) d x=e^{-x_{0}}\left(x_{0}+1\right) \approx x_{0} e^{-x_{0}}=\frac{D}{k T} e^{-D / k T}
$$


Therefore, the number of ordinary molecular collisions required per dissociation, is

$$
z_{d}=\frac{1}{C_{d} F\left(x_{0}\right)}=\frac{1}{c_{d}} \frac{e^{x_{0}}}{x_{0}}
$$

where $C_{d}$ is the efficiency of the collisions between molecules of sufficient energy in producing dissociation. The reciprocal relaxation time for dissociation is

$$
\omega_{\mathrm{d}} \approx \frac{\mathrm{N}}{\mathrm{Z}_{\mathrm{d}} \mathrm{d}} .
$$

where $\mathrm{N}$ is the number of collisions per second ( $c f .2 .18,2.18 \mathrm{a}$ ). The mean free path for dissociation is (cf.2.24).

$$
\lambda_{\mathrm{d}}=\mathrm{y} / \boldsymbol{\omega}_{\mathrm{d}}
$$

Because of the large factor $e^{x_{0}}$, the number of collisions required for dissociation is very large at temperatures of $3000-5000^{\circ} \mathrm{K}$ at which the dissociation of air becomes important (Tables II and III). In Table VII (cf.p. 52a) we give the values of $z_{3}, \boldsymbol{\omega}_{a}$, and $\boldsymbol{\lambda}_{a}$ for oxygen and nitrogen; it is seen that $z_{d}$ lles between $10^{6}$ and $10^{12}$ and is thus much higher than for the vibration. It is obvious that impurities cannot greatly affect $Z_{\mathrm{d}}$ because the decisive factor is the Boltzmann factor rather than the efficlency of the collisions.

\section{Conclusions on the Excitation of Alr.}

Vibrations

Neither the theory nor the available experimental results are sufficiently accurate to permit any quantitative predictions on the mean free path for the vibrations of the most important gases, $\mathrm{O}_{2}$ and $\mathrm{N}_{2}$. It is certain that these mean free paths are rather long, and under certain circumstances they may become comparable with the dimensions of a projectile. 
Experimental information is available (Table V) only on the effect of $\mathbb{N}_{2}$ and of water vapor on the vibration of $\mathrm{O}_{2}$; in addition an upper IImit is known for the effect of $\mathrm{O}_{2}$ on the $\mathrm{O}_{2}$ vibration. The effect of $\mathrm{N}_{2}$ on $\mathrm{O}_{2}$ is described by an effective range $s=3 \cdot 10-9 \mathrm{~cm}$ as computed in $(2.18 \mathrm{a})$. In Table IV, we have calculated $\mathrm{Z}$ as a function of temperature with this value of $\mathrm{s}$. These calculated values should represent the temperature dependence of the effect of $\mathrm{N}_{2}$ on the vibrations of $\mathrm{O}_{2}$ fairly accurately, 1.e., within a factor of perhaps 3.

For the collisions between two $\mathrm{O}_{2}$ molecules, the experiments give $\mathrm{z}>500,000$ at room temperature. Assuming $\mathrm{z}=10^{6}$, we obtain $\mathrm{s}=4^{\circ} 10^{-9} \mathrm{~cm}$ from (2.13, 15). The values of $\mathrm{z}$ for $\mathrm{O}_{2}$ at various temperatures with $\mathrm{s}=4^{\circ} 10^{-9}$ are also given in Table IV; the actual $\mathrm{Z}$ for $\mathrm{O}_{2}-\mathrm{O}_{2}$ collisions may be smaller than the values given in the table by about a factor of 2 , but greater by any amount. In any case, in air the vibrations of $\mathrm{O}_{2}$ will be excited much more easily by colIisions with $\mathrm{N}_{2}$ than by collisions with other $\mathrm{O}_{2}$ molecules, both beceuse of the greater abundance of $\mathrm{N}_{2}$ and of the smaller $\mathrm{Z}$.

In Table VI we give the estimated times and distances required to establish equilibrium of the molecular vibretions in air. In particular, Table VI A gives the reciprocal time of relaxation, $\omega_{0}$, as a function of temperature for various assumptions. $w_{0}$ has been calculated from $(2.18,18 a$ and 22$)$, considering $q$ as independent of the temperature. The values of $q$ were obtained by comparing the values of $\mathrm{Z}$ and $\omega_{0}$ given by Kneser and Kunze for room temperature; these $q^{8} \mathrm{~s}$ seem somewhat low but the errors are not important compared with the uncertainties in the theory. The density of the air was assumed to be $1.18^{\circ} 10^{-3}$, corresponding to atmospheric pressure at $300^{\circ} \mathrm{K}$. In the first row of Table VI A, $W_{0}$ is given for the vibrations of $\mathrm{O}_{2}$, taking into account only the collisions with $\mathrm{N}_{2}$ molecules. The neglected collisions with $\mathrm{O}_{2}$ can increase $\omega_{0}$ by 10 per cent at most.

Collisions with water molecules are very effective in exciting the vibrations of $\mathrm{O}_{2}$ (Table V). Four hundred collisions with $\mathrm{H}_{2} \mathrm{O}$ are sufficient for dem. excitation of $\mathrm{O}_{2}$ at room temperature; therefore, as little as one per cent of water 
vapor will be three times as effective as all the nitrogen in the air. In the second row of Table VI A we have listed the value of $\omega_{0}$ for collisions of $\mathrm{O}_{2}$ molecules with $\mathrm{H}_{2} \mathrm{O}$, assuming a concentration of 1 per cent water vapor by volume. On hot humid days, the water concentration may be easily 3 per cent. It is seen that, at $I$ per cent, the collisions with $\mathrm{H}_{2} \mathrm{O}$ are more effective than those with $\mathrm{N}_{2}$ at $500^{\circ} \mathrm{K}$, but less effective at $700^{\circ}$ and higher temperatures. This behavior is due to the fact that $\omega_{0}$ rises very rapidly with temperature for collisions with $N_{2}$, but rather slowly for collisions with $\mathrm{H}_{2} \mathrm{O}$. (Table IV)

While the information obtainable on $\mathrm{O}_{2}$ is fairly satisfactory, very little can be said about the excitation of the vibrations of $\mathrm{N}_{2}$. It is reasonable to expect that the effect of $\mathrm{O}_{2}$ on the $\mathrm{N}_{2}$ vibrations can be described by the same effective range, $s=3 \cdot 10^{-9} \mathrm{~cm}$, as the effect of $\mathrm{N}_{2}$ on $\mathrm{O}_{2}$, although this is by no means certain. In Table IV we have given the corresponding $\mathrm{Z}$ for $\mathrm{N}_{2}$; it is much higher than the $\mathrm{Z}$ for $\mathrm{O}_{2}$ with the same $\mathrm{S}$ because the vibration erequency of $\mathrm{N}_{2}$ is about 50 per cent higher than for $0_{2}$. The third row of Table VI gives $\omega_{0}$ for the excitation of the vibration of $\mathrm{N}_{2}$ in air, assuming that only collisions $\mathrm{w}$ with $\mathrm{O}_{2}$ are effective. These values for $\omega_{0}$ can therefore be regarded as lower limits.

No experimental results are available concerning the effect of collision with the $\mathrm{N}_{2}$ molecules on the vibration of $\mathrm{N}_{2}$. To obtain any theoretical estimate, we must find an interpretation of the difference between the effective range $s$ for $\mathrm{o}_{2}-\mathrm{O}_{2}$ and $\mathrm{O}_{2}-\mathrm{N}_{2}$ collisions, $\mathrm{viz} . \mathrm{s} \approx 4 \cdot 10^{-9}$ and $\mathrm{s}=3 \cdot 10^{-9} \mathrm{~cm}$, respectively。 There are two possible interpretations: The first alternative is to assume that generally collisions between different molecules are more effective than between equal ones. Such a tendency seems to exist in the experimental result (Table V) but there appears to be no theoretical justification. Moreover, it is to be remembered that the experiments were mostly done with polyatomic molecules, for 
which there are other reasons for a stronger interaction (cf. point 2 in the section on Experimental Results of par. 28). Thus we do not get an explanation fof the effectiveness of the collisions between $\mathrm{O}_{2}$ and $\mathrm{N}_{2}$, and we are led to the second alternative. This is based on the fact that $\mathrm{N}_{2}$ is a more compact molecule than $\mathrm{O}_{2}$, having a greater binding energy and smaller distance between the atoms. From this difference in structure we may expect a shorter range of the forces for $\mathrm{N}_{2}$ which would explain the smaller value of $\mathrm{s}$ for $\mathrm{O}_{2}-\mathrm{N}_{2}$ collisions as compared with $\mathrm{O}_{2}=\mathrm{O}_{2}$ 。

If this second alternative is accepted, we should expect an even smaller $s$ for the interaction between two $\mathrm{N}_{2}$ molecules than for the $\mathrm{N}_{2}-\mathrm{O}_{2}$ interaction. We have therefore included in Table IV the values of $\mathrm{Z}$ for $\mathrm{N}_{2}$ obtalned with $\mathbf{s}=2.5 \cdot 10^{-9} \mathrm{~cm}$. These values are, of course, considerably smaller than for $\mathrm{N}_{2}$ and $s=3 \cdot 10^{-9} \mathrm{~cm}$, and not much larger than for $\mathrm{O}_{2}$ and $\mathrm{s}=3 \cdot 10^{-9} \mathrm{~cm}$. In the fifth row of Table VI A we have given $\omega_{0}$ for $\mathrm{N}_{2}$ in air, assuming $\mathrm{s}=2.5 \cdot 10^{-9} \mathrm{~cm}$ for the interaction $\mathrm{N}_{2}-\mathrm{N}_{2}$; the values thus obtained are only slightly less than those for $\mathrm{O}_{2}$ (first row).

On the other hand, if the first alternative explanation above is assumed, the interaction between two $\mathrm{N}_{2}$ molecules would have a large $s$, just as the interaction between two $\mathrm{O}_{2}$ molecules. In this case, the $\mathrm{N}_{2}-\mathrm{N}_{2}$ collisions would not contribute appreciably to the excitation of $\mathrm{N}_{2}$ vibrations, and $\omega_{0}$ for $\mathrm{N}_{2}$ would be given by the third line in Table VI $A$ in which the $\mathbb{N}_{2}-0_{2}$ collisions alone are taken into account.

Finally, as a compromise, we have also given the results when $s=3 \cdot 010^{-9}$ $\mathrm{cm}$ is assumed to be valid for collislons between two $\mathrm{N}_{2}$ molecules as well as between $\mathrm{N}_{2}$ and $\mathrm{O}_{2}$ (fourth row of Table VI A).

The effect of water vapor on $\mathrm{N}_{2}$ is also unknown。 $\mathrm{H}_{2} \mathrm{O}$ is extremely effective in exciting the vibrations of $\mathrm{O}_{2}$ and $\mathrm{CO}_{2}$ (cf. Table V) as well as of $\mathrm{N}_{20}$, $\mathrm{CS}_{2}$ and $\operatorname{COS}$ (Knudsen and Fricke, Ioc.cit。). By pure analogy we might therefore 
conclude that it would also be effective on $\mathrm{N}_{2}$, and we have therefore included in Tabie IV a calculation of $Z$ for collisions between $\mathrm{N}_{2}$ and $\mathrm{H}_{2} \mathrm{O}$, assuming the same $\mathrm{s}$ as for collistons between $\mathrm{O}_{2}$ and $\mathrm{H}_{2} \mathrm{O}$ but taking into account the higher 2 of $\mathrm{N}_{2}$. However, it must be remembered that $\mathrm{O}_{2}$ has a chemical affinity to $\mathrm{H}_{2} \mathrm{O}$ while $\mathrm{N}_{2}$ has very little; therefore, collisions with weter may be much less effective on $\mathrm{N}_{2}$ (2 higher) than is indicated by the last line of Table IV.

In Table VI A, last row, we have computed $\omega_{\circ}$ for collisions between $\mathrm{N}_{2}$ and $\mathrm{H}_{2} \mathrm{O}$, assuming $I$ per cent water vapor in air of denstty $1.18 \cdot 10^{-3}$, and assuming the $Z$ as given in Table IV. Presumably, these values of $\omega_{0}$ are on the high side. Whether or not the humidity has an appreciable influence on the vibrations of $\mathrm{N}_{2}$, depends not only on the temperature and on the correctness of our assumption about the interaction between $\mathrm{N}_{2}$ and $\mathrm{H}_{2} \mathrm{O}$, but also on the assumed interaction between $N_{2}$ and $N_{2}$. If the latter is strong $\left(s=2.5 \cdot 10^{-9}\right)$, the humidity is rather unimportant even at low $\mathrm{T}$; if it is weak (collisions with $\mathrm{O}_{2}$ only), the humidity is the decisive factor. This again indicates the extreme uncertainty of the data on the excitation of the vibration of $\mathrm{N}_{2}$.

In Table VI B, we have calculated the mean free path for vibration, $\lambda_{\mathrm{V}}$, on the high pressure side of a shock wave produced in "standard air", i.e., when the temperature and pressure on the low pressure side are $300^{\circ}$ and 1 atmosphere respectively. $\lambda_{\mathrm{V}}$ is given in Table VI as a function of the temperature $\mathbb{T}_{3}$ which is obtained on the high pressure side at large distance from the front of the shock wave (par.3); $T_{3}$ again is a known function of the velocity $v_{1}$ of the shock wave (Tole VIII). Velocity and density on the high pressure side were also taken from Table VIII (par.3), the asymptotic values $v_{3}$, $\rho_{3}$ being used. The so defined $\lambda_{\nabla}$ is related to the $\omega_{0}$ given in Table VI A by

$$
\lambda_{v}=\frac{v_{3}}{\omega_{0}} \frac{\rho_{1}}{\rho_{3}}
$$


Table VI. Relaxation Time and Mean Free Path of Vibration for $\mathrm{O}_{2}$ and $\mathrm{N}_{2}$ in Air.

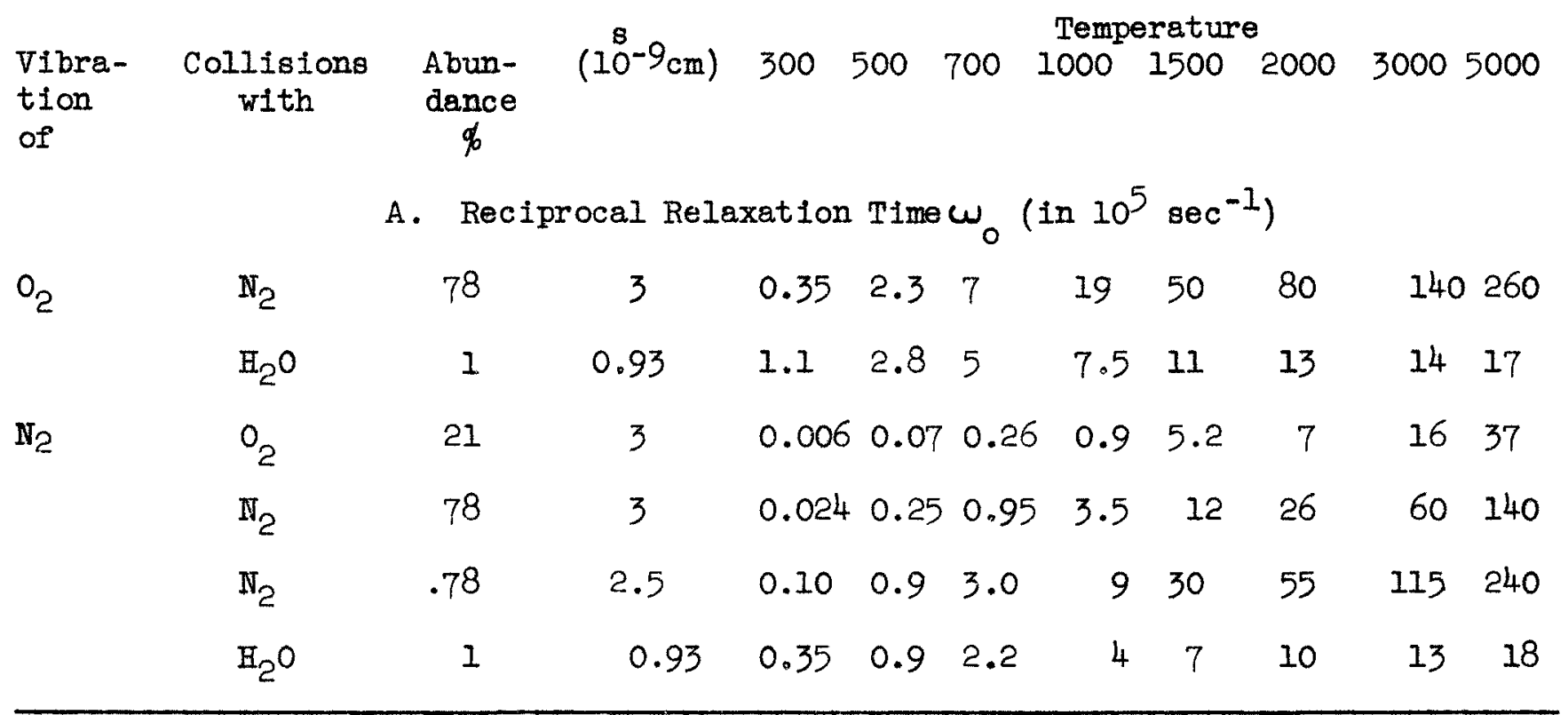

B. Mean Free Path of Vibration $\lambda_{V}$ (in millimeters)

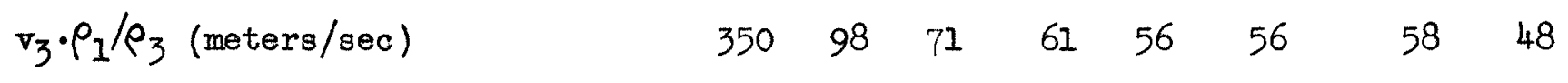

Vibra- Collisions with

tion of
$\mathrm{O}_{2} \quad \mathrm{~N}_{2}$ only
$\begin{array}{lllllllllllll}10 & 0.43 & 0.10 & 0.032 & 0.011 & 0.007 & 0.004 & 0.0018\end{array}$
$\mathrm{N}_{2}$ and $\mathrm{H}_{2} \mathrm{O}(1 \%)$
$\begin{array}{lllllllll}2.4 & 0.19 & 0.06 & 0.023 & 0.009 & 0.006 & 0.003 & 0.0017\end{array}$
$\begin{array}{llllllllllll}\mathrm{N}_{2} & \mathrm{O}_{2} \text { only } & 550 & 14 & 2.7 & 0.7 & 0.18 & 0.08 & 0.036 & 0.013\end{array}$
$\mathrm{O}_{2}$ and $\mathrm{N}_{2}\left(\mathrm{~s}=3 \cdot 10^{-9} \mathrm{~cm}\right) \quad 110 \quad 3.0 \quad 0.6 \quad 0.14 \quad 0.037 \quad 0.0170 .00750 .0027$
$\mathrm{O}_{2}$ and $\mathrm{N}_{2}\left(\mathrm{~s}=2.5 \cdot 10^{-9} \mathrm{~cm}\right) \quad 33 \quad 1.0 \quad 0.22 \quad 0.06 \quad 0.017 \quad 0.009 \quad 0.004_{5} 0.0017$

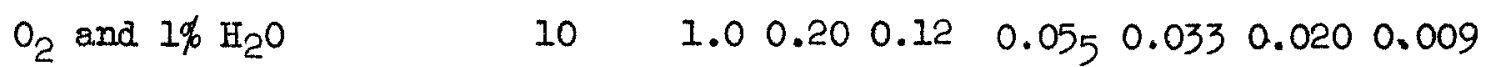
$\mathrm{O}_{2}, \mathrm{~N}_{2}\left(\mathrm{~s}=3 \cdot 10^{-9}\right)$ and $\mathrm{H}_{2} \mathrm{O} 9.5 \quad 0.8 \quad 0.21 \quad 0.07 \quad 0.025 \quad 0.013 \quad 0.006_{5} \quad 0.0025$
$\mathrm{O}_{2}, \mathrm{~N}_{2}\left(\mathrm{~s}=2.5 \cdot 10^{-9}\right)$ and $\mathrm{H}_{2} \mathrm{O} 8 \quad 0.5 \quad 0.13 \quad 0.010 .014 \quad 0.008 \quad 0.004 \quad 0.0016$


The quantity $\nabla_{3}: \rho_{1} / \rho_{3}$ is given in the first row of Table VI $B$, in meters per second. Then $\lambda_{V}$ is given for various assumptions.

The value of $\lambda_{\mathrm{v}}$ for $\mathrm{O}_{2}$ in dry air decreases from 10 millimeters at $300^{\circ}$ to $1 / 150$ millimeter at $2000^{\circ}$ and $1 / 500$ at $5000^{\circ}$. If the initial pressure is low-let us say, $1 / 100$ atmosphere-- $\lambda_{V}$ is proportionally greater (100 times) and may there fore ea olly reach considerable values. In wet air containing $I$ per cent of water vapor by volume, $\lambda_{v}$ for $\mathrm{O}_{2}$ is reduced to $2 \mathrm{~mm}$ at $300^{\circ}$ but is almost the same as for dry air when $\mathrm{T} 1500^{\circ} \mathrm{K}$.

For the vibrations of nitrogen, the value of $\lambda_{\mathrm{V}}$ is extremely uncertain. If the collisions with $\mathrm{N}_{2}$ are unimportant (cf. above) and if the air is dry, only collisions with $\mathrm{O}_{2}$ need to be considered; then $\lambda_{\mathrm{v}}$ ls as large as half a meter at $300^{\circ} \mathrm{K}$. Since the vibrational energy of $\mathrm{N}_{2}$ becomes important only for $\mathrm{T} 600^{\circ}$ (Table II), $\lambda_{V}$ is important only at higher temperatures; but even at $T=700^{\circ}$ we obtain $\lambda_{v} \approx 3$ if only collisions with $\mathrm{O}_{2}$ are effective. On the other hand, if collisions with $N_{2}$ are very effective $\left(\mathrm{s}=2.5 \cdot 10^{-9} \mathrm{~cm}\right), \lambda_{\mathrm{v}}$ is reduced by about a factor of 12 at $700^{\circ}$, and a factor of 8 at $5000^{\circ}$. If collisions with $\mathrm{H}_{2} \mathrm{O}$ are as effective as assumed in Table IV and VI A, a water vapor content of 1 per cent reduces $\lambda_{V}$ by factors varying from 9 to 1.05 when only temperatures $\geqslant 700^{\circ}$ are considered.

Apart from the uncertainties in the assumptions, there is also an uncertainty in the Landau-Teller theory itself which makes the temperature dependence of $\lambda_{\nabla}$ uncertain by a factor of about 3 even if $\omega_{0}$ at room temperature is accurately known.

The unsatisfactory state of our knowledge about the mean free path for molecular vibrations in air could be improved by experiments on the dispersion and absorption of sound in mixtures of $\mathrm{O}_{2}$ and $\mathrm{N}_{2}$ of varying composition and free from impurities. Such experiments should be done at $T \approx 700^{\circ} \mathrm{K}$ or higher in order to ensure sufficient excitation of the molecular vibrations of $\mathrm{N}_{2}$. With such experiments available, the dependence of $\lambda_{\mathrm{V}}$ on the temperature at higher tempertures 
could probably be calculated from the Iandau-Teller theory with fair accuracy. The influence of humidity should also be investlgated experimentally.

Nitrogen and oxygen are probably almost unique in their large valuee of $\mathrm{z}$, and therefore of $\lambda_{\mathrm{V}}$. Other molecules have much lower frequencies or much greater chemical activity (cf. above). Therefore for most other gases, and especially for complicated polyatomic ones (explosives!), $\lambda_{V}$ will in general be too small to be of any practical importance.

Dissociation. In Table VII, we give the number of molecular collisions $z_{\mathrm{d}}$ required for one dissociation process, the reciprocal time of relaxation $\omega_{\mathrm{d}}$, and the mean free path $\lambda_{d}$ for dissoclation. These quantities were calculated from Eqns. (2.28, 29 and 29a). The constants $N$ and $v$ were assumed as in Table VI, namely $\mathbb{N}=5 \cdot 10^{9} \mathrm{sec}^{-1}$ at $300^{\circ} \mathrm{K}$. and one atmosphere, and proportional to $\sqrt{\mathrm{T}}$ otherwise; $v$ equal to the velocity $v_{3}$ of air on the high pressure side of a shock wave produced in "standard air"; $\propto$ was taken from Table II, $C_{d}$ was arbitrarily put equal to $1 / 100$. $\mathrm{z}_{\mathrm{d}}$ is, of course, independent of the pressure; $\omega_{\mathrm{d}}$ is calculated for a density* of $1.18 \cdot 10^{-3}$ as in Table VI, and $\lambda_{\alpha}$ for the actual conditions on the high pressure side of a shock wave. It can be seen that the mean free paths obtained are very long indeed, decreasing for $\mathrm{O}_{2}$ from a little under one meter at $2500^{\circ}$ to a little over one millimeter at $5000^{\circ}$. Therefore we should expect large effects from lack of dissociation equilibrium in shack waves which are sufficiently violent to produce dissoclation. We must emphasize again the great uncertainty of the figures in Table VII which is caused by the lack of knowledge of $C_{d}$. Here again experiments would be desirable but they seem considerably more difficult than in the case of vibrations. Possibly studies of the dissociation equilibrium of other gases (e.g.s $\mathrm{N}_{2} \mathrm{O}_{4}$ ) would help.

* This is not quite consistent since $\alpha$, which occurs in (2.29), was calculated for an 8 times larger density, but $p=1.18 \cdot 10^{-3}$ was chosen for comparison with the $w_{0}$ in Table VI. 
Table VII. Relaxation Time and Mean Free Path for the Dissociation of Air

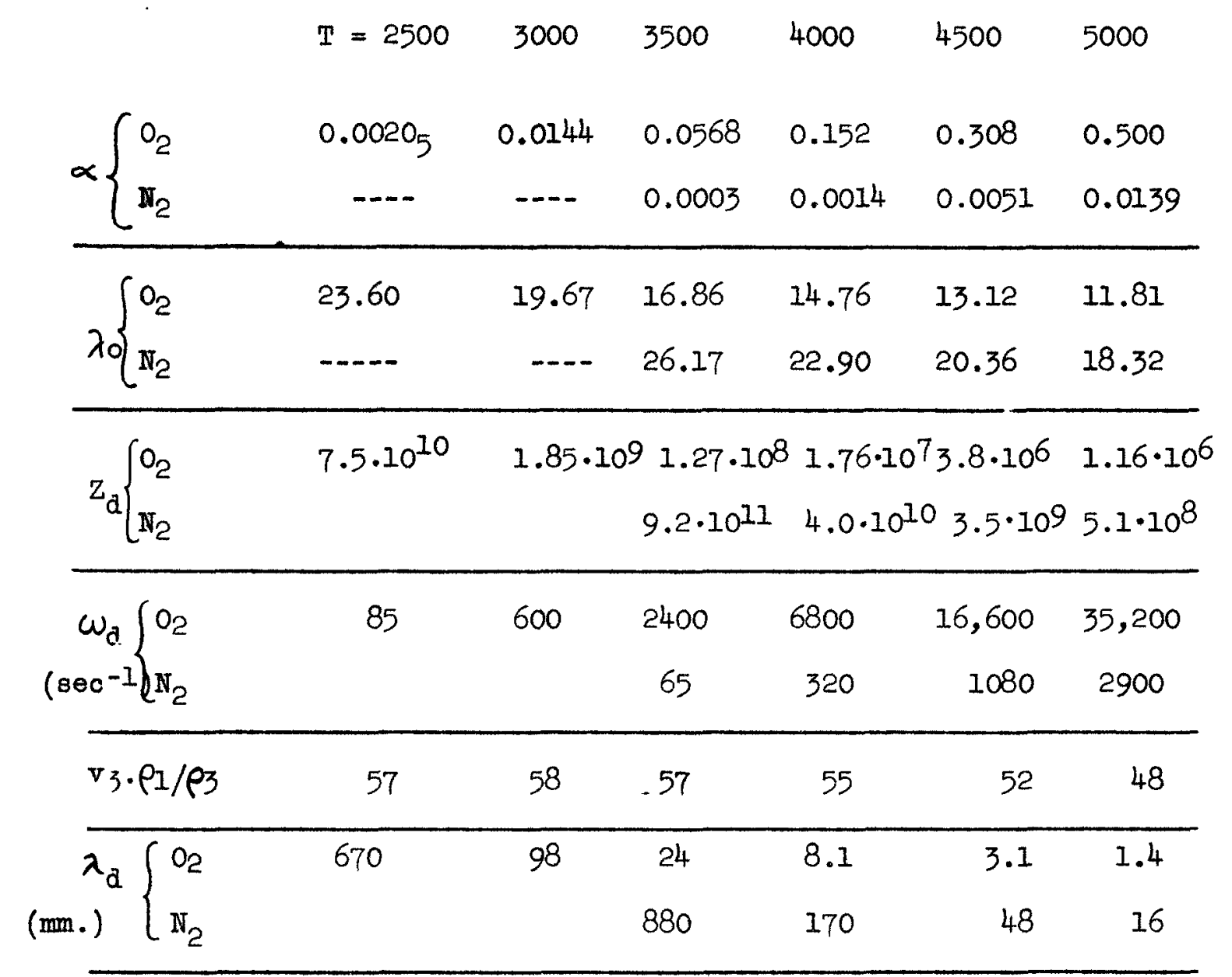




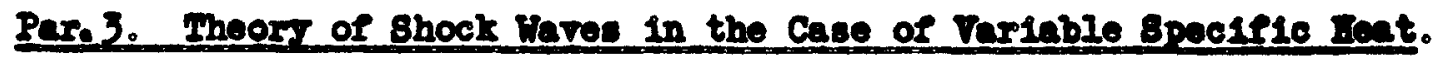

\section{Eotat1on:}

We shall denote by letters without subecripte the plowical quantitios at ang point in the shock wave, by letters with the subecript 1 the quantities on the lor proseure side of the wave, by the subseript 2 those on the migh preseure B Ide immodiately at the front on the vare, and by the subscript 3 those on the high preseure side at large distance from the vave front, 1.0., whore equilibrium bus been establishod for vibrations and dissociation. Wo ball also use $\mathrm{h}$ and 1 for arbitrary polnts on the hich and low proseure elde, roppoctively. Wo consider the onedinopical case throughout. For further notation, see the end of the 1ntroduction.

\section{rundemental Iquations:}

1. Iquation of continuits

$$
\text { Pr = convtant }=
$$

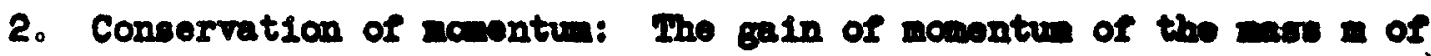
ges, $=\nabla_{g}$ is equal to the decrease in pressure, -3p. Thereforew

$$
\begin{gathered}
p+m v=\text { constant }=v T \\
(\text { depinition of } \nabla)
\end{gathered}
$$

3. Conservation of ener BS

$$
P+\frac{R}{P}+\frac{1}{2} v^{2}+\text { constant }=\frac{1}{2} c^{2}
$$

When the ga flow adiabatically into vacuun, $\eta / p$ and therofore also I 80 to 20 ro so that $v$ approaches $c$. Introducing pdefined in $(1.3),(3.3)$ becomes

$$
\beta \frac{p}{\rho}+\frac{1}{2} \nabla^{2}=\frac{1}{2} c^{2}
$$

has been extensively discussed in par. $I$ and 2 . It has been shown in par. 2 that $\beta$ dopends on the existence or non-existence of oquilibriun botween the various degabes

* It Is intoresting that in our case $p+\rho v^{2}$ is constant while in the incouprossIble fluid it is $\mathrm{g}+(1 / 2) \mathrm{v}^{2}$. In both conservetion laws, tho elosontary law is $d p+\rho v d r=0$, but for the integation we mat assume $\rho=$ constant in the incampressible liuid, $p r=$ constant in our case. $\quad$., 31 
of freedom of the molecules. If there is equilibrim, $\beta$ is a function of the tomperature (or $p / p$ ) alone; tables of $\beta$ for this case are given in par. I for nitrogen, oxygen and eif (Tables II and III). Equilibrium will exist erergmere on the lor pressure side of the shock vare $\left(\beta=\beta_{1}\right)$ and asynptotically at largo distance from the vare fropt on the high preseure side $\left(\beta=\beta_{3}\right)$. The value of $\beta$ on the bigh prossure side imediately at the vare fropt $\left(\beta_{2}\right)$ can be celculated easily from the fact that the energy content of the Inert degrees of freedom (ribration, excitation and dissocietion) is the same as on the low pressure olde (ci. begining of pars 4). In the particular case when the temperature on the lon prensure eide ia low onough so that there is no appreciable onergy in the inert degreos of rspedon (Iulfilled for air below $\left.400^{\circ} \mathrm{K}\right)_{8}$ we have simply $\beta_{2}=\beta_{1}(=7 / 2$ for dintanic enees). In the intermediate region on the high pressure side, $\beta$ mut be considered as varying from $\beta_{2}$ to $\beta_{3}$ in a way wich will be discussed in pars 4 . For the moment, we oball consider $\beta$ as given and determine the othor physical quantitios from it. The three constants $m_{g} \nabla$, and $c$ defined in $(3.1,2,3)$ are civan by the preseure, density, and relocity of the inconing bee on the low presenre side:

$$
\begin{gathered}
\nabla=\frac{P_{1}}{P_{1}{ }^{v}}+v_{1} \\
c^{2}=v_{1}^{2}+2 p_{1} \frac{P_{1}}{P_{1}}
\end{gathered}
$$

It is often convenient to introduce the velocity of sound by putting

$$
p h=e^{2} / \gamma
$$

(Valid only in the absence of dissociation)

In most practical applications, the temperature on the low preseure side is eupIleiontly low so that $\mathrm{dp/ar}=0$ and (cr.1.19, 1.21)

$$
\gamma=\frac{\beta}{\gamma-1}
$$


If this is true, wo may rowrite (3.4):

$$
\begin{aligned}
& \nabla=v_{1}+\frac{\beta_{1}-1}{\beta_{1}} \frac{a_{1}^{2}}{v_{1}} \\
& c^{2}=v_{1}^{2}+2\left(\beta_{1}-1\right) a_{1}^{2}
\end{aligned}
$$

Solution of Fundamental Equations:

$(3.1)$ and (3.2) may be used to eliminate $p$ and $p, \nabla i z$ 。

$$
\begin{aligned}
& p=m / v \\
& p=m(\nabla-\nabla) \\
& \frac{p}{\rho}=(\nabla-\nabla) \nabla
\end{aligned}
$$

These equations are important to calculate $\rho_{2} p_{2}$ and $T$ once $v$ bas been determined. Inserting now (3.9) into $(3.30)$, wo find:

$$
\beta(\nabla-\nabla) v+\frac{1}{2} \nabla^{2}=\frac{1}{2} c^{2}
$$

and therefore

$$
\nabla=\frac{\beta \nabla \pm \sqrt{\beta^{2} \nabla^{2}-(2 \beta-1) c^{2}}}{2 \beta-1}
$$

If $\nabla$ and $c$ are given, there are, for any value of $\beta$, two solutions for $\nabla$. In general, these two solutions are real (for oxcoption, cf, par, 5 , p. 71); If real, they are both positive. It can easily be ohow that the lareor value of $v$ [plus sign in $(3.20)]$ in general is greater than the corresponding velocity of sound, the smaller $v$ smaller than the corresponding $a_{0}$

To show this, wo calculate from $(3,9)$

$$
\stackrel{p}{\rho}=\frac{\gamma}{2 \beta-1}\left[(\beta-1) \nabla+\sqrt{\beta^{2} \nabla^{2}-(2 \beta-1) c^{2}}\right]
$$


where the upper and lower sign correnpond to the upper and lover sien in $(3.10)$. How if we assune that $\beta$ does not depend much on the temperature, we nas use (3.3. 6) and have

$$
a^{2}=\gamma \frac{\gamma}{\rho}=\frac{\gamma}{2 \beta-1}\left[\beta+\frac{\rho}{\beta-1} \sqrt{\beta^{2} \gamma_{-}(2 \beta-1) e^{2}}\right]
$$

This sive

$$
\nabla=\frac{a^{2}}{\nabla}=\mp \frac{1 \cdots}{\rho-1} \sqrt{\rho^{2} p^{2}-(2 \rho-1) c^{2}}
$$

1. o. Ta for the upper, rea for the 10wer, siga.

In roality, $\beta$ doos depend on $i$ and therefore (3.6) is not corrset but should be roplaced by (cr. 1.19)

$$
\gamma=\frac{\rho+d \alpha / d \log I}{\beta-1+d / d \log T}
$$

(essuning no discociation).

In all practical cases $\rho$ increases with temperature so that $\gamma$ (and thorefore $a^{2}$ )

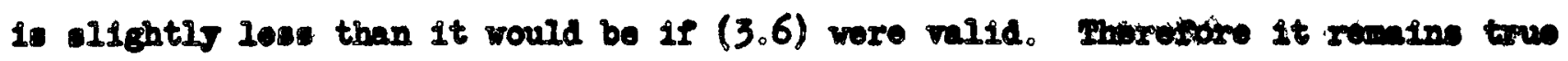

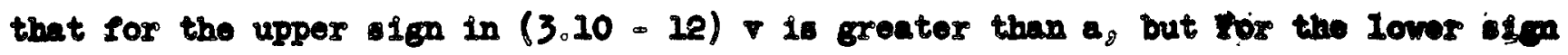

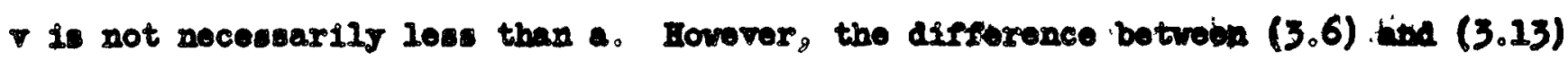

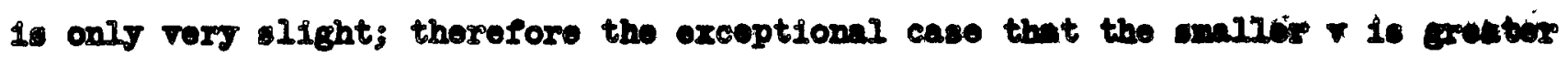
than the corresponding a will bo of ninor importance. $A$ more detalled diacuselen will bo given in par. $5, p \circ 80,81$.

\section{Diecuesion:}

On the 10 pressure oide of the shock wate, the velocity will vo given by (3.10) with the plue sign (in the folloring denoted by the subveript 2 , for 10w), on the high pressure ide by the colution with the ninus sien (oubseript $h$ for high). No have pointed out above that $\beta$ will have the equilibriun valuos, 
$\beta_{1}$ and $\beta_{3}$, both on the low pressure side and on the high pressure side at large distance from the wave front. The equilibrium value of $\beta$ is a unique function of the temperature so that $\beta_{1}$ and $\beta_{3}$ are completely determined by $\mathrm{T}_{1}$ and $\mathrm{T}_{3}$. Let us denote by $T_{e}(\beta)$ the temperature which belongs to a given $\beta$ in thermal equilibrium; it is the function tabulated in Tables II and III and it increases monotonically with $\beta$. On the other hand, the theory of shock waves (Eq.3.10) gives $v_{3}$ uniquely in terms of $\beta_{3}$ since $V$ and $c$ are given by the initial conditions (cf. $3.4 \mathrm{a}, \mathrm{b}$ ) and the sign of the square root is also determined (negative). From $v_{3}$ in turn $\mathrm{T}_{3}$ is determined through (3.9)* so that $T$ is, by the shock wave theory, a given function of $\beta$ which we shall denote by $T_{8}(\beta)$. The temperature $T_{3}$, and the energy content $\beta_{3}$, are determined by solving the equation

$$
T_{s}(\beta)=T_{e}(\beta)
$$

It can easily be shown that this equation has only one solution for which the velocity $v$ is smaller than the corresponding velocity of sound, 1.e., only one "high pressure" solution. In most cases, this follows from the fact that $\mathbb{T}_{8}(\beta)$ decreases with increasing $\beta$ over almost the whole range of $\beta$ (cf. $3.16 c$ and especially par.6) whereas $T_{e}(\beta)$ increases monotonically. In the small range of $\beta$ in which $\mathrm{T}_{s}(\beta)$ increases (cf. par.6), this increase is slower than that of $\mathrm{T}_{e}$; in fact, the condition $\mathrm{v}<\mathrm{a}$ is equivalent with $\frac{d \mathrm{~T}_{\mathrm{8}}}{\mathrm{d} \beta}<\frac{\mathrm{dT}}{\mathrm{d} \beta}$.

We may conclude, then, that $\beta_{3}, T_{3}$ and of course also the other physical quantities $\left(p_{3}, \rho_{3}, \nabla_{3}\right)$ are uniquely determined by the initial conditions $p_{1}, \rho_{1}$, $v_{1}$. In other words, all the physicel quantities $(v, p, \rho, T$, etc.) on the high pressure side at sufficlent distance from the shock wave are independent of all Intervening processes connected with the establishment of equilibrium between 
"Inert" and "active" degrees of freedom. Therefore, if the dimensions of the obstacle causing the shock vave are large compared. With the mean free path for $v 1$ bration, etc. (par. 2), the pressure, resistance, etc. will be the same as if all

degrees of freedom were in equilibrium all the time.

$\therefore$ We courd rewrite $I q .(3.10)$ inserting the values $(3.4 a, b)$ or $(3.7 a, b)$

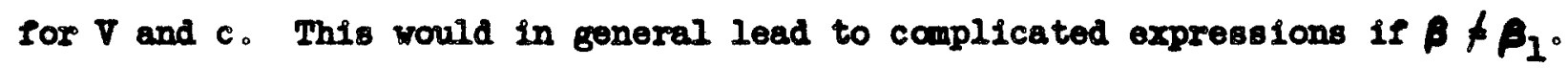
However, simple results are obtained in the two cases (a) $\beta=\beta_{1}$ and $(b) v_{1} \gg a_{1}$.

a) For $\beta=\beta_{1}$ and $(\alpha \varphi / \alpha \log T)_{1}=0$ our theory reduces to the usual theory of shock waves, and $(3.10)$ bocomes (use $3.7 a, b^{8}$ )

$$
v=\frac{v_{1}+(\beta-1) \frac{a_{1}^{2}}{v_{1}}+(\beta-1)\left(v_{1}-\frac{a_{1}^{2}}{v_{1}}\right)}{2 \beta-1}
$$

which gives

$$
\begin{aligned}
& \nabla_{l}=v_{1} \\
& v_{h}=\frac{1}{2 \beta-1}\left[\nabla_{1}+2(\beta-1) \frac{a_{1}^{2}}{v_{1}}\right]
\end{aligned}
$$

(3.15) is the fundamental equation of the usual theory in an especially convenient Porm。

b) Simple expressions can also be obtained if $v_{1} \gg a_{1}$ no matter whether $\boldsymbol{\beta}=\boldsymbol{\beta}_{1}$ or not. In this case (cf. $3.4 a_{9}$ b) we have $v \approx c \approx \nabla_{1}$ and $(3.10)$ gives

$$
v_{h}=\frac{v_{1}}{2 \beta-1}
$$

From $(3.8,9)$ we find then

$$
\begin{gathered}
P_{h}=P_{1}(2 \beta-1) \\
P_{h}=P_{1} \nabla_{1}^{2} \frac{2(\beta-1)}{2 \beta-1}
\end{gathered}
$$




$$
\left(\mathrm{RP}_{\mathrm{h}}=\right) \quad \frac{\mathrm{P}_{\mathrm{h}}}{P_{\mathrm{h}}}=v_{1}^{2} \frac{2(\beta-1)}{(2 \beta-1)^{2}}
$$

The relative error of these formulae is about $2 \beta_{1} a_{1}{ }^{2} / v_{1}{ }^{2}$ (for $p_{h}$ only $a_{1}{ }^{2} / v_{1}{ }^{2}$ )。 In the approximation used here, the quantities on the high pressure side are independent of $\beta_{1}$ and depend only on the local value of $\beta$ on the high pressure side. It is seen that the value of $\mathrm{Ph}_{\mathrm{h}}$ increases linearly with $\beta$; in the special case $\rho=7 / 2$ (diatomic gases with translation and rotation only) $(3.16 a)$ gives the, vell-know result that the density in a shock wave can only increase six-fold. since $\beta$ increase considerably at high temperatures, the actual increase of $\rho$ can be wuch greater than six-fold. $p_{h}$ depends only olightly on $\beta$ (for large $\beta$ ) be cause, in our limit $v_{1} \rightarrow a_{1}$ we have $v_{h}$ er $v$ and therefore $p_{h}$ is approximately m (ce. $3.8 b$ ) which is a constant。

Finally, $T_{h}$ decreases* strongly with increasing $\beta$ because the total

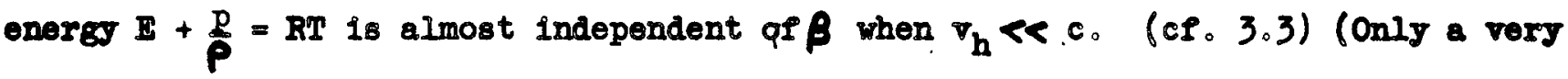
small amount of kinetic energy is lefti). As an example, we compare the asjmptotic velues of the physifgil quantities for air at ordinary temperatures $(\beta=7 / 2)$ and for hot air with the vibrations fully excited but no dissociation $(\beta=9 / 2)$.

\begin{tabular}{ccc}
\hline & $\boldsymbol{\beta}=7 / 2$ & $\boldsymbol{\beta}=9 / 2$ \\
\hline$\nabla_{\mathrm{h}} / v_{1}$ & $1 / 6$ & $1 / 8$ \\
$P_{\mathrm{h}} / \boldsymbol{P}_{1}$ & 6 & 8 \\
$\boldsymbol{P}_{\mathrm{h}} / \boldsymbol{P}_{1}{ }_{1}{ }^{2}$ & $5 / 6=0.833$ & $7 / 8=0.875$ \\
$\mathrm{RT}_{\mathrm{h}} / \mathrm{v}_{1}{ }^{2}$ & $5 / 36=0.239$ & $7 / 64=0.109$ \\
\hline
\end{tabular}

- In case of dissociation $(3.16 \mathrm{c})$ holds for $\operatorname{RI}_{h}\left(1+\sigma_{h}\right)$ rather than for $\operatorname{RI}_{h}$. * This statement holds also in case of qissociation because T is a monotonic sunction of $\mathrm{p} / \mathrm{p}$. 
Table:

Table VII gives the physical characteristice on the high preseure eide of a shock wave produced in air of $300^{\circ}$ relvin (27 Centigrade) by incident streams of varlous velocities. For the construction of auch a table, it is convenlent to consider the temperatures $T_{1}$ and $T_{3}$ as given and to celculate $v_{1}$ and $v_{3}$, rather than to start from $r_{2}$ and $v_{1}$ and caloulate $g_{3}, v_{3}$ from them. $\rho_{3}$ is a tebulated function of $\mathrm{I}_{3}$ (cr. Table III). Eqe (3.3) and (3.9) way be rewritten

$$
\cdots \quad \begin{aligned}
\frac{P_{3}}{P_{3}} \cdot \frac{1}{v_{3}}+\nabla_{3} & =\frac{p_{1}}{P_{1}} \cdot \frac{1}{v_{1}}+v_{1} \\
& 2 \beta_{3} \frac{p_{3}}{3}+v_{3}^{2}=2 \beta_{1} \frac{P_{1}}{P_{1}}+v_{1}{ }^{2}
\end{aligned}
$$

Bolving for $\nabla_{1}, \nabla_{3}$ wo obtain

$$
\frac{\nabla_{1}}{\nabla_{3}}=b+\cdots \sqrt{b^{2}+\frac{p_{1} / P_{1}}{p_{3} / \rho_{3}}}
$$

and

$$
\frac{v_{3}^{2}}{p_{3} / \rho_{3}}=\frac{2 b^{2}+1-b_{2} P_{3} / p_{3} p_{1}}{\left(v_{1} / \nabla_{3}\right)^{2}-1}
$$

where

$$
b=\beta_{3}-\frac{1}{2}-\frac{p_{1} / P_{1}}{p_{3} / P_{3}} \quad\left(\beta_{1}-\frac{1}{2}\right)
$$

These formulae are oultable for compution.

Table VII gives the important physicel quantities as functions of $v_{1} / a_{1}$, the ratio of the velocity of the Inccuing strean to the corresponding velocity of sound, for values of $\nabla_{1} / a_{1} \operatorname{srcm} 1.5$ to 11.6 . Volocity, density, temperature and pressure on the high pressure side are given both at large distance from the wave front (subscript 3) and immodiately at the vave front (subscript 2). The latter 
Table VIII. Characteristics of Shock Waves in Air

\begin{tabular}{lllllllll}
\hline$v_{1} / \mathrm{a}_{1}$ & $v_{3} / \mathrm{a}_{1}$ & $v_{2} / \mathrm{a}_{1}$ & $\rho_{3} / \rho_{1}$ & $\rho_{2} / \rho_{1}$ & $\mathrm{~T}_{3}$ & $\mathrm{~T}_{2}$ & $\mathrm{p}_{3} / \mathrm{p}_{1}$ & $\mathrm{p}_{2} / \mathrm{p}_{1}$ \\
\hline 1.523 & 0.798 & 0.800 & 1.907 & 1.904 & 400 & 400 & 2.543 & 2.538 \\
1.984 & 0.746 & $0.749_{5}$ & 2.659 & 2.647 & 500 & 501 & 4.432 & $4.42_{5}$ \\
2.377 & 0.737 & 0.47 & 3.225 & 3.189 & 600 & 604 & 6.450 & $6.41_{5}$ \\
2.725 & 0.744 & 0.759 & 3.663 & 3.591 & 700 & 709 & 8.547 & 8.49 \\
3.041 & $0.757_{5}$ & 0.779 & 4.015 & 3.904 & 800 & 816 & 10.707 & 10.62 \\
3.331 & $0.77_{5}$ & $0.803_{5}$ & 4.314 & 4.146 & 900 & 925 & 12.94 & 12.77 \\
3.611 & $0.795_{5}$ & 0.831 & 4.540 & 4.346 & 1000 & 1036 & 15.23 & 15.01 \\
4.235 & $0.835_{5}$ & 0.900 & 5.069 & 4.706 & 1250 & 1320 & 21.12 & 20.72 \\
4.797 & 0.880 & 0.970 & 5.454 & 4.945 & 1500 & 1616 & 27.27 & 26.66 \\
5.307 & $0.923_{5}$ & 1.041 & 5.746 & 5.098 & 1750 & 1925 & 33.52 & 32.7 \\
5.778 & $0.966_{5}$ & $1.103_{5}$ & 5.978 & 5.236 & 2000 & 2222 & 39.85 & 38.75 \\
6.643 & 1.045 & $1.228_{5}$ & 6.359 & 5.409 & 2500 & 2848 & 3.01 & 51.4 \\
7.453 & 1.115 & 1.350 & 6.685 & 5.521 & 3000 & 3510 & 67.05 & 64.6 \\
8.315 & 1.1675 & 1.481 & 7.122 & 5.615 & 3500 & 4300 & 84.09 & 80.5 \\
9.297 & 1.208 & $1.633_{5}$ & 7.697 & 5.691 & 4000 & 5300 & 106.02 & 100.6 \\
10.410 & $1.241_{5}$ & 1.809 & 8.385 & 5.754 & 4500 & 6570 & 134.40 & 126.2 \\
11.595 & 1.269 & 1.997 & 9.136 & 5.804 & 5000 & 8030 & 168.38 & 155.6
\end{tabular}


guantities were calculated assuming that the Inert degrees of freedom retain the same energy as on the low pressure ide. Since this energy is practicelly zero, we can put $\beta_{2}=\beta_{1}=3.483$ (cf. Table III), and can therefore calculate $\nabla_{2}$ from (3.15) (and the remaining quantities from $(3.8,9)$ ).

Comparing the quantities with subscripts 2 and 3 , we find approximate agreement up to about $v_{1} / a_{1}=3$. At higher $v_{1} / a_{1}$, we find that $p_{3}$ is considerably greater than $p_{2}$ (cf. $3.16 a$ ) and, correspondingly (beceuse of the continuity equation) $v_{3}<v_{2}$. Thus the shock wave consists of a discontinuous compression folloved by a sradual further compression which extends over a distance determined by the conalderations of par. 2 and, 4.: Along withe the strong increase of the density thero Is a swall increase of the pressure from $p_{2}$ to $p_{3}$ (last two columns, cf. also $3.16 \mathrm{~b})$, but even if the discontinuous change of the pressure is by as much as a factor of 100, the following continuous one 18 only 5,4 per cent. Therefore, as far as the pressure 1s concerned, the change of $\beta$ with temperature is rather unimportant. The temperature increases discontinuously at the wave front from $T_{1}=300^{\circ}$ to $T_{2}$ and then decreaseg* gradually to $T_{3}$, due to a transfer of energy from the "active" degrees of freedon by whose excitetion the temperature is defined, to the "Inert" degrees. The temperature decrease 18 greatest for the ; highest $v_{1} / a_{1}$ where 1 is is from over 8000 to 5000 degrees.

Of some interest are perhaps the columns $v_{2} / a_{1}$ and $v_{3} / a_{1}$. It is seen that for relatively small $v_{1}$, the velocities $\dot{v}_{2}$ and $v_{3}$ are amaller than the veloc-: Ity of sound on the low pressure side, $a_{1}$, and that they decrease with increasing $v_{1}$. Then a minimum is reached and at still larger values of $v_{1}$, the high pressure velocities $v_{2}$ and $v_{3}$ become greater than $a_{1}$. For $v_{2}$, the existence of a minimum can be seen directly from (3.15); the minimum 18 obtained for

* For very "Boft" shock waves ( $\nabla_{1}$ only slightly greater than a ) there can be a light increase from $\mathrm{T}_{2}$ to $\mathrm{T}_{3}$, ct. par. 6 . 


$$
\cdot \frac{v_{1}}{a_{1}}=\sqrt{2\left(\beta_{1}-1\right)}=2.23 \quad(\text { for } \beta=3.483)
$$

and has the value

$$
\frac{v_{2 \text { min }}}{a_{1}}=\frac{2 \sqrt{2\left(\beta_{1}-1\right)}}{a_{1}-1}=0.747(\text { for } \beta=3.483)
$$

For very high $\nabla_{1}$ we obtain

$$
\begin{aligned}
& \nabla_{2}=\frac{\nabla_{1}}{2 B_{1}-1} \\
& \nabla_{3}=\frac{\nabla_{1}}{2 \beta_{3}-1}
\end{aligned}
$$

Par. 4. The Approach of Equilibrium.

We may assume that the energy of the inert degrees of freedom (vibration, etc.) does not change discontinuously at the front of the shock wave while that of the active degrees of freedom does. It will therefore be convenient to split the total energy $E$ into the part due to translation and rotation, $E_{a}$ (a=active) and the part due to vibrations, electronic excitation and dissociation, $E_{1}$ $(1=$ inert $)$. We put

$$
\mathrm{E}_{\mathrm{a}}+\frac{\mathrm{p}}{\mathrm{p}}=\mathrm{B}_{\mathrm{a}} \stackrel{\mathrm{p}}{\mathrm{p}}
$$

where $\beta_{a}$ is practically constant and equal to $7 / 2$ for diatomic gases (5/2 for monatomic ones). Further, we must have

$$
E_{11}=E_{12}
$$

1.e., the energy of the inert degrees of freedom is the same on both sides of the shock wave front. Then (3.3a) becomes:

$$
\beta_{a} \frac{p}{\rho}+E_{i}+\frac{1}{2} \nabla^{2}=\frac{1}{2} c^{2}
$$


Instead of $(3.9 a)$ we have

$$
\beta_{a}(\nabla-\nabla) \nabla+\frac{1}{2} \nabla^{2}=\frac{1}{2} c^{2}-B_{1}
$$

and Instead of $(3.10)$ :

$$
r=\frac{\beta_{a} \pm \sqrt{\beta_{a}^{2} p^{2}-\left(2 \beta_{a}-1\right)\left(o^{2}-2 B_{1}\right)}}{2 \beta_{a}-1}
$$

Iquation (3.4a) 18 unchanged:

$$
v^{\prime}=\frac{P_{1}}{P_{1} \nabla_{2}}+\nabla_{1}
$$

ville $(3.6 b)$ is replaced by

$$
c^{2}=v_{1}^{2}+2 \beta_{a} \mathrm{RT}_{1}+2 \mathrm{~F}_{11}
$$

It can be seen essily that the phrsical quantities $P_{2}, P_{2}, \nabla_{2}, T_{2}$ on the high pressure olde immodiateify at the front of the shock wave are exactly as if the moleculax vibretion vere absent entirely. We may use Fq. (3.15) with $\beta$ a Instead of $\beta$ to calculate $\nabla_{2}$, and 'then obtein the other quantities from $(3.8,9)$. This has actually been done in Table VII.

Parther in the high pressure reglon, the inert degrees of freedon 121 gradually come into equilibrium. If wo bave only one such degree, $0.8 .$, the vibration (subscript v), we may urito (par. 2)

$$
\frac{d E_{v}}{d x}=\frac{B_{v}(T)-E_{v}}{\lambda_{v}(T, \rho)}
$$

where $T$ is the Iocal temperature-(delined as $p / p R), F_{v}^{\prime}(T)=\beta_{\nabla}(T) p / \rho$ the oqut11brium value of the vibrational eneres corrosponding to $T, F$ the ectual $100 a l$ value of the vibrational energy, $x$ the coordinate perpendicular to the vave frant counted Irom the low pressure to the high pressure ide, and $\lambda_{\mathrm{v}}$ the moen Iree 
path for vibrations (par, 2) which will depend on the local density and temperature. similar equations, but with a different $\lambda_{8}$ will hold for dissociation and excitat10n.

To intograte (4.7), it is more convenient to calculato $x$ as a function of the phreical variables than to do the reverse. The integration must in in general be done numerically because $\lambda_{\nabla}$ and $F_{\nabla}{ }^{8}$ are given only by numerical tables (rablec II to VII) and depend in a compliceted way on the variable T. A dependence of $B_{\nabla}$ and $\lambda_{\checkmark}$ on the density does not present ans additional difficulty because $p$ 1s, by $(3.8 a)$ and $(3.9)$, a unique function of $\mathrm{p} / \mathrm{p}=\mathrm{RT}$. Assuming again that vibration is the only inert degree of freedom (true for alr below $2500^{\circ} \mathrm{K}$ ), I can be celculated in terms of T from $(4.3)$, eliminating $\nabla$ by use of $(3.8 \mathrm{~b})$ :

$$
E_{\nabla}=\frac{1}{2} c^{2}-\frac{1}{4} \nabla^{2}-\left(\beta_{a}-\frac{1}{2}\right) R t+\frac{1}{4} v \sqrt{\nabla^{2}-4 \mathrm{RT}}
$$

This is a fairiy complicated dependence. It seoms hardly worth while to carry out numerical integrations of $(4.7),(4.8)$ for apecial cases.

1 However, it is ealy to estinate the distance required to establish equilibrium. It must be of the order of $\lambda_{v^{g}}$ more precisely of the largest velue of $\lambda_{\nabla}$ occurring, $1_{0} \theta_{0}$, the one corresponding to the lovest temperature (par, 2) existing on the high pressure olde of the shock vave Oxdinarily (1.0, with the exception of the case discussed in par, 6), this lowest temperature is reached

* If there are sereral inert degrees of freedom (ribration, exc1tation, dissociation, possibly of several gases), there is one equation of the tjpe (4.7) for each of them. The unknowns are the energies $\mathbf{F}_{1}(1), \mathbf{F}_{\mathrm{f}}\left({ }^{2}\right)$, etc. In the varlous inert degrees of freedcm, and $I_{2}$ the temperature as defined by the eneres in the active degrees of freedom. Irom the temperature $\nabla_{g} P_{1}$ and $p$ can be determined,

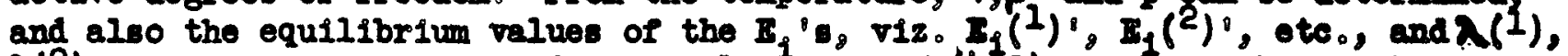
$\mathcal{N}^{2}$ ), etc. The number of differential equations $(4.7)$ is obviousis one less then the number of unknowns. The system is completed by $\mathrm{Iq}(4.8)$, w1th $\mathrm{F}$ replaged by $\mathbf{E}_{1}=\mathbf{E}_{1}(\mathbf{I})+\mathbf{z}_{1}(\mathbf{2})+\ldots$ 
in the equilibrium state (highest $\beta$, cf. $3.16 \mathrm{c}$ ). Therefore it is only necessary to read from Tables VI, VIII the $\lambda$ for the temperature $\mathrm{T}_{3}$ and pressure $\mathrm{p}_{3}$.

The integration is simple if $\mathrm{T}_{2}, \rho_{2}$ are sufficiently close to the values $T_{3}, P_{3}$. Then we may consider $\lambda$ as constant and $T[$ according to (4.3)] and therefore $\mathrm{E}_{\nabla}$ (according to par.l) as depending linearly on $E_{\mathrm{V}}$, viz.

$$
\frac{d E_{v^{\prime}}}{d E_{v}}=-\mu=\text { constant }
$$

$\mu$ is positive because $T$, and therefore $\mathrm{E}_{\mathrm{V}}$ ', decreases with increasing $\mathrm{E}_{\mathrm{V}}$ (cf. 4.8). Using (4.9), (4.7) integrates immediately to

$$
E_{v}^{\prime}(T)-E_{v}=\left[E_{v^{\prime}}\left(T_{2}\right)-E_{v l}\right] e^{-x(1+\mu) / \lambda\left(T_{3}\right)}
$$

[remembering (4.2)!] . Thus the deviation from equilibrium decreases exponentially as we go away from the wave front. Since all physical quantities are expected to change very little ( $\mathrm{T}_{2} \approx \mathrm{T}_{3}$, etc.), $\mathrm{T}, \rho, \mathrm{p}, \nabla$, etc. are sufficiently nearly linear functions of $\mathrm{E}_{\mathrm{v}}{ }^{\prime}-\mathrm{E}_{\mathrm{V}}$, so that

$$
T(X)-T_{3}=\left(T_{2}-T_{3}\right) e^{-x(I+\mu) / \lambda}
$$

and similarly for the remaining quantities.

It need hardy be pointed out that the gradual change of the physical quantities occurs only on the high pressure side because the gas streams from the low pressure to the high pressure side if we consider the wave front as fixed. Increasing $\mathrm{x}$ means therefore a later time. On the low pressure side, the wave front is sharp (except in the case of par.5) because any molecules which may cross the wave front against the stream, i.e., from high pressure to low pressure side, will soon revert to the high pressure side because of collisions. There will therefore be no perturbation of the state on the low pressure side outside of a distance of a few times the ordinary mean free path from the wave front. 
The stability of the shock wave against diffusion is insured by the fao't that the gas velocity relative to the shock wave is greater than the velocity of sound on the low pressure side, less on the high pressure side. If there should at any time be a splitting of the shock wave into two parts (due to a small obstacle or so), these two parts will soon reunite: suppose a small disturbance runs before the main shock wave; then its velocity will be the velocity of sound, $a_{1}$, and it will be overtaken by the shock wave of velocity $v_{1}$. If the small disturbance runs behind, Its velocity (relative to the gas) will be $a_{3}$ and It will therefore catch up with the main shock wave which mores only with the velocity $v_{3}$ relative to the high pressure gas.

Par. 5. Diffuse Shock Waves.

In this and the following section, we shall discuss some peculiar phenomena which occur only for very "soft" shock waves, 1.e., when the velocity $v_{1}$ of the shock wave is only slightly greater than the velocity of sound, $a_{1}$. These two sections are in no way important for the general problem of shock waves in a medium of variable specific heat which has been solved in par. 3 and 4 . Especially for a substance like air, whose inert degrees of freedom are very little excited at room temperature, the effects discussed in par. 5 and 6 have no practical significance but only academic interest. In the two sections, wo solve some mathematical difficulties which might occur if the formulae of par. 3 and 4 were applied indiscriminately, and complete some proofs which were left incomplete in per. 3 and 4. The most importent of these is the proof (end of par. 5) that for anj initial conditions $p_{1}, p_{1}, T_{1}, \nabla_{1}$ there is always exactly one solution of the shock wave equations in thermal equilibrium for which $\nabla$ is less than the velocity of sound a (high pressure solution) and one for which $v>a_{8}$ the latter being identical with the initial conditions. 
In particular, in par. 5 we shall discuss the case when the velocity $v_{I}$ of the shock wave is less than that velocity of sound, $a_{a l}$, which is obtained when only the specific heat of the active degrees of freedom is considered. Such shock waves are possible because the "active velocity of sound", al, is greater than the ordinary velocity of sound; we have

$$
\begin{aligned}
& a_{a 1}^{2}=\frac{p_{1}}{\rho_{I}}\left(1+\frac{R}{c_{\text {val }}}\right) \\
& a_{1}^{2}=\frac{p_{1}}{\rho_{I}}\left(1+\frac{R}{c_{v l}}\right)
\end{aligned}
$$

where $c_{v I}$ is the total specific heat at constant volume on the low pressure side of the shock wave while $c_{\text {val }}$ is the specific heat of the active degrees of Ireedom alone. Since $c_{v}>c_{v a}$, we have $a_{a l}>a_{1}$, and therefore there are values of $v_{1}$ such that

$$
a_{1}<v_{1}<a_{a l}
$$

These values of $v_{1}$ shall be the subject of the investigations of this section. The difficulty is the following: according to the general theory, a shock wave must exist if $v_{1}>a_{1}$. On the other hand, the front of the shock wave behaves as if only the active degrees of freedom existed (par.4), therefore the wave front cannot exist if $v_{1}<a_{a l}$. Mathematically, this difficulty appears in the form that the square root in (4.5) becomes imaginary if the constants $c, V$ and $\mathrm{E}_{11}$ are inserted which correspond to the initial conditions. Practically, the region determined by $(5.2)$ is very narrow. If $c_{v l}$ and $c_{\text {val }}$ are not too different, we have

$$
\frac{a_{a l}}{a_{1}}=1+\frac{R\left(c_{v 1}-c_{v a l}\right)}{2 c_{v a l}\left(c_{v 1}+R\right)}
$$


Taking $c_{\text {val }}=\frac{5}{2} R$ (diatomic molecule) and even assuming $c_{v l}$ to be as large as 3R; this gives only 1.025. For air at $300^{\circ} \mathrm{K}, c_{\nabla I}-c_{v a l} i_{0} \theta_{\circ}$, the specific heat of the vibration, is about $0.007 \mathrm{R}$; then $a_{a 1} / a_{1}=1.0004$ so that the interval (5.2) is exceedingly narrow.

The solution of the difficulty which we found above for the velocity interval (5.2) is as follows: there exists a shock wave which is propagated without change of shape, but in which the velocity goes continuously through the velocity of sound. The extension of this shock wave in spece is again of the order of the mean free path for vibration, $\lambda_{v}$ (cf。par.4); and the variation of the physical quantities with $x$ is again determined by (4.7) in conjunction with $(4.5),(3.8),(3.9)$. However, instead of having a continuous variation only on the high pressure side (lower sign in $(4.5)$ ), we now have it also on the low preso sure side. Coming from the latter, wo have a gradual increase of temperature, density and pressure together with a gradual increase of the vibrational energy $E_{V^{*}}$ The connection between $\forall$ and $E_{V}$ is given by the positive sign in (4.5)。 The change of the temperature is such that the vibrational energy falls more and more short of its equilibrium value, or mathematically, the difference $\mathbb{E}_{\mathbf{V}}^{r}(T)-\mathbb{R}_{\nabla}$ $\left(\operatorname{or} \beta^{\circ}-\beta\right)$ increases (cr。5.21)。 Thereby the square root in $(4.5)$ is reduced until it vanishes. From then on, the negative sign must be taken with the square root; there is a further gradual increase of $T_{2} p$ and $p$ but now the vibrational energy "catches up" again with its equilibrium value $\mathbb{E}_{\mathrm{v}}{ }^{0}(\mathrm{~T})$ which It reaches at large distance from the shock wave.

For the quantitative treatment, we introduce that value $\beta_{0}$ of $\beta$ at which the square root in $(3.10)$ vanishes when $\nabla$ and $c$ are kept constant. $\beta_{0}$ is. thus a function of the initial conditions of the shock vave. The temperature, pressure, etc., which are obtained by setting $\beta=\beta_{0}$ in $(3.10)$, will be denoted by $p_{0}, p_{0}$, etc.

Obriously, $\beta_{0}$ is defined by

$$
\beta_{0}{ }^{2} \nabla^{2}=\left(2 \beta_{0}-1\right) c^{2}
$$


which has the solution*

$$
\rho_{0}=\frac{c}{v^{2}}\left(c+\sqrt{c^{2}-\nabla^{2}}\right)
$$

$\beta_{0}$ is thus uniquely determined by the initial conditions $v_{1}, p_{1}, P_{1}$ and $\beta_{1}$ (ce. 3.4a, b).

The square root in $(3.10)$, divided by $\nabla$, may now be written

$$
\sqrt{\beta^{2}-\frac{\beta_{0}^{2}}{2 \beta_{0}-1}(2 \beta-1)}=\sqrt{\left(\beta-\beta_{0}\right) \cdot\left(\beta-\frac{\beta_{0}}{2 \beta_{0}-1}\right)}
$$

Neglecting all higher terms in $\beta=\beta_{0}$ this gives

$$
\sqrt{\beta-\beta_{0}} \cdot \sqrt{\frac{\beta_{0}\left(\beta_{0}-1\right)}{\beta_{0}-\frac{1}{2}}}
$$

Inserting this into (3.10) and neglecting again all higher porers of than the square root (for more accurate formula, see (6.9a)), we get

$$
\frac{v}{\bar{v}}=\frac{1}{2 \beta_{0}-1}\left[\beta_{0} \pm \sqrt{\beta-\beta_{0}} \sqrt{\frac{\beta_{0}\left(\beta_{0}-1\right)}{\beta_{0}-\frac{1}{2}}}\right]
$$

Denoting the velocity for $\beta=\dot{\beta}_{0}$ by $\nabla_{0}$, this gives

$$
\frac{\nabla}{\nabla_{0}}=1 \pm \sqrt{\frac{\beta_{0}-1}{\beta_{0} \beta_{0}-\frac{1}{2}}} \cdot \sqrt{\beta-\beta_{0}}
$$

8imilarly, wo get from (3.11) for the temperature (assuning no dissociation):

$$
\frac{T}{T_{0}}=1+\sqrt{\frac{\beta_{-} \beta_{0}}{\beta_{0}\left(\beta_{0}-\frac{1}{2}\right)\left(\beta_{0}-1\right)}}
$$

* The negatire aige before $\sqrt{c^{2}-v^{2}}$ would lead to a value of $\beta$ emaller than unity wich cannot be attained by the physical quantity $\beta(\beta=5 / \beta$, cr. par. 1$)$. 
where $\mathrm{T}_{0}$ is the temperature corresponding to $\beta_{\circ}$, which is given by (cf. $3.9,5.6$ )

$$
\mathrm{RT}_{0}=\frac{\beta_{0}-1}{\beta_{0}} \quad \mathrm{v}_{0}{ }^{2}
$$

Introducing the abbreviation

$$
y=\frac{T}{T_{0}}-1
$$

we have from $(5.7)$

$$
\beta-\beta_{0}=\beta_{0}\left(\beta_{0}-\frac{1}{2}\right)\left(\beta_{0}-1\right) y^{2}
$$

and from $(5.6 a)$

$$
\frac{v}{v_{0}}-1=-\left(\beta_{0}-1\right) y
$$

We consider three velocities of sound, viz.

(1) the velocity of sound with the active degrees of freedom alone.

$$
a_{a}=\sqrt{\gamma a^{R T}}=\sqrt{\frac{\beta_{a}}{\beta_{a}-1} R T}
$$

(2) the true velocity of sound

$$
a=\sqrt{\gamma \mathrm{RT}}
$$

where (cf. 1.19)

$$
\gamma=\frac{\beta+d \beta / d \log T}{\beta-1+d \beta / d \log T}
$$

If $\beta$ changes not too rapidly with $T$, this may be written:

$$
\gamma=\frac{\beta}{\beta-1}-\frac{\alpha \beta / d \log T}{(\beta-1)^{2}}
$$


(3) the expression

$$
a^{i}=\sqrt{\frac{\beta}{\beta-1} \mathrm{RT}}
$$

which would result if $\alpha \beta / \alpha \log T$ were neglected in $(5.10 a)$. Ordinarity theo three velocities of sound are quite close to each other so that we may write, neglecting higher powers of $\alpha \beta / \alpha \log T$ and of $\beta-\beta_{a}$ :

$$
\begin{aligned}
& \frac{a_{a}}{a^{b}}=1+\frac{\beta-\beta a}{2 \beta(\beta-1)} \\
& \frac{a}{a^{\prime}}=1-\frac{d \beta / \alpha \log T}{2 \beta(\beta-1)}
\end{aligned}
$$

From $(5.7 a)$ and $(5.11)$ we see that

$$
\nabla_{0}=a_{0}{ }^{0}
$$

Since $a^{\prime}$ varies as $\sqrt{T}$, we have therefore from $(5.8 a)$

$$
\frac{v}{a^{8}}-1=-\left(\beta_{0}-\frac{1}{2} y\right)
$$

Therefore the value of $\mathrm{y}$ at which $\mathrm{v}$ is equal to the actual velocity of sound, 18 (cf.5.12b)

$$
y_{m}=\frac{d \beta / d \log T}{2 \beta_{0}\left(\beta_{0}-\frac{1}{2}\right)\left(\beta_{0}-1\right)}=B
$$

The value of $y$ at which $v$ is equal to $a_{a}$, is (cfo $\left.5.12 a\right)$

$$
y_{a}=-\frac{\beta_{0}-\beta_{a}}{2 \beta_{0}\left(\beta_{0}-\frac{1}{2}\right)\left(\beta_{0}-1\right)}=-A
$$


Fior $\mathrm{y}_{1}<\mathrm{y}_{\mathrm{a}}$, there will be an ordinary shock wave with wave front. Only for $y_{a}<y_{1}<y_{m}$ there will be a diffuse shock wave $\left(y_{1}=\right.$ initial value of $y$ on low pressure slde). From $(5.14)$ and (5.15), we have *

$$
\frac{v}{a}-1=\left(\beta_{0}-\frac{I}{2}\right)(B-y)
$$

It is convenient to introduce the abbreviation

$$
z=\frac{v_{I}-a_{I}}{a_{a I}-a_{I}}=\frac{B-y_{I}}{B+A}
$$

Diffuse shock waves will be obtained for $z$ between 0 and 1 .

Since the local value of $\beta$ is given by $(5.8)$ for all $y$, we may write

$$
\beta-\beta_{1}=\beta_{0}\left(\beta_{0}-\frac{1}{2}\right)\left(\beta_{0}-1\right)\left(y^{2}-y_{1}^{2}\right)
$$

On the other hand, the equilibrium value of $\beta$ which we denote by $\beta^{8}$, as in (4.7), may be regarded as a linear function of $J$ in the small temperature interval considered, and since $\beta_{1}^{\prime}=\beta_{1}$, we have:

$$
\beta^{i}-\beta_{1}=\mathrm{d} \beta / \mathrm{d} \log \mathrm{T} \cdot\left(\mathrm{y}-\mathrm{y}_{1}\right)
$$

Asymptotically on the high pressure side we must have equilibrium again so that $\beta_{3^{\prime}}=\beta_{3}$. Comparing $(5.18)$ and $(5.19)$, we find

$$
\beta_{0}\left(\beta_{0}-\frac{1}{2}\right)\left(\beta_{0}-1\right)\left(y_{3}^{2}-y_{1}^{2}\right)=(\alpha \beta / d \log T)\left(y_{3}-y_{1}\right)
$$

Therefore (cf.5.15)

$$
\mathrm{y}_{3}=2 \mathrm{~B}=\mathrm{y}_{1}
$$

* Eq. (5.16a) contains the solution of the problem discussed after Eq (3.13). The vanishing of the square root $\left(\beta_{0}, T_{0}, v_{0}\right)$ actually does not represent the point where $v=a$, but this point lies at slightly higher temperature and lower velocity (1.e. in the region described by the lower sign of the square root in (3.10)). 
This means that the temperature at which $v$ is equal to the velocity of sound (cf.5.15) is the average of the initial and final temperature. For the final temperature, we have always $v<$ a as we might expect. We can now discuss the actual equation (4.7) describing the change of the physical quantities in the shock wave." $\mathrm{Eq}$ (14.7) may be re-written

$$
\frac{d}{d x}\left[T\left(\beta-\beta_{a}\right)\right]=\frac{1}{\lambda}\left(\beta^{i}-\beta\right) T
$$

Using $(5.8),(5.18),(5.19)$ and neglecting higher powers of $\beta-\beta_{0}$, this gives

$$
\begin{aligned}
& \left(\beta_{0}-\beta_{a}\right) \frac{d y}{d x}+\beta_{0}\left(\beta_{0}-\frac{1}{2}\right)\left(\beta_{0}-1\right) 2 y \frac{d y}{d x} \\
= & \frac{1}{\lambda} \frac{d \beta}{d \log T}\left(y-y_{1}\right)-\beta_{0}\left(\beta_{0}-\frac{1}{2}\right)\left(\beta_{0}-1\right)\left(y^{2}-y_{1}{ }^{2}\right)(5.21 a)
\end{aligned}
$$

With $(5.15,16,20)$ this becomes

$$
\frac{(A+y) d y}{\left(y-y_{1}\right)\left(y_{3}-y\right)}=\frac{d x}{2 \lambda}
$$

Elementary integration gives

$$
\frac{A+y_{1}}{y_{3}-y_{1}} \log \left(y-y_{1}\right)-\frac{A+y_{3}}{y_{3}-y_{1}} \log \left(y_{3}-y\right)=\frac{x}{2 \lambda}
$$

Using again (5.20) and (5.17), this may be written

$$
(1-z) \log \left(y-y_{1}\right)-(1+z) \log \left(y_{3}-y\right)=\frac{x z}{\lambda}
$$

The temperature approaches $T_{1}$ asymptotically for large negative $x_{8} T_{3}$ for large positive $x_{0}$. The approach in each case is exponential, viz.

$$
\begin{aligned}
& T-T_{1} \exp \left(\frac{x z}{(1-z)}\right) \text { for } x \rightarrow-\infty \\
& T_{3}-T \cdot \exp \left(-\frac{x z}{(1+z)}\right) \text { for } x \rightarrow+\infty
\end{aligned}
$$


Thus, for $z \neq 0$, the approach of the asymptotic value is more rapid on the low temperature side $\left(T_{1}\right)$ than on the high temperature one $\left(T_{3}\right)$. In the limit $z \rightarrow 1$, 1.e. when $v$ approaches the "active" velocity of sound, a $a_{\text {al }}$ the diffuse shock wave automatically goes over into a wave with a discontinuous front as we might expect.

In the other limiting case $z \rightarrow 0,1 . e ., \nabla_{1} \rightarrow a_{1}$, the shock wave becomes symmetrical and more extended; (5.23) is then equivalent to

$$
T=\frac{1}{2}\left(T_{1}+T_{3}\right)+\frac{1}{2}\left(T_{3}-T_{1}\right) \frac{e^{x z / \lambda}-1}{e^{x z / \lambda}+1}
$$

1.e., the extension of the shock wave is of the $\operatorname{order} \lambda / \mathrm{z}$.

The formulae of this section can also be applied to the approach of rotational equilibrium if $v_{1}$ is smaller than the velocity of sound, $a_{t l}$, which would be obtained if the translation alone is considered in the specific heat. In this case, (5.23) Gives the distribution of temperature (defined by the translational enerEJ): where $\lambda$ is the mean free path for rotation which, of course, is very small (par, 2A). Aside from giving the solution for shock wave velocities between $a_{1}$, and $a_{a l}$, this section completes two proofs which were left incomplete in par. $3:$

1. On the high pressure side of a shock wave, the gas velocity $v_{3}$ is always smaller than the velocity of sound a3. To show this, we calculate, instead of (3.12), the value of $a^{2} / v$ with the correct value (3.13) of $\gamma$. If we use (5.10a) (In which higher powers of ap/d $\log T$ have been neglected), we obtain for the lower sign

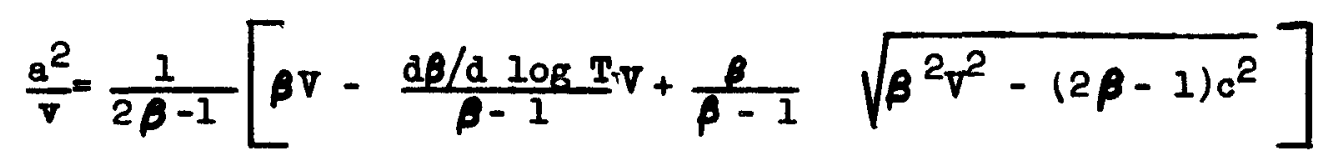

Subtracting this from (3.10) (again with the lower sign), we find

$$
v-\frac{a^{2}}{\nabla}=\frac{v}{\beta-1}\left[\frac{\alpha \beta / \alpha \log T}{2 \beta-1}-\sqrt{\beta^{2}-(2 \beta-1) \frac{c^{2}}{\nabla^{2}}}\right]
$$


In order that this be negative (1.e. $v_{3}<a_{3}$ ), we must have (cf. 5.5a, 5.4)

$$
\beta-\beta_{0}>\frac{(\alpha \beta / \alpha \log T)^{2}}{4 \beta_{0}\left(\beta_{0}-\frac{1}{2}\right)\left(\beta_{0}-1\right)}
$$

The value of $\beta-\beta_{0}$ on the right hand side is sufficiently small so that $(5.6 a)$, (5.7) and all subsequent formulae are valid (cf. 6.18). If $\beta-\beta$ o is equal to the right hand side of (5.28), it follows from (5.7), (5.15) that $\mathrm{y}$ is just equal to $B$.

Th1s result coincides with (5.15) where we have shown that $v$ is just equal to the velocity of sound a for $y=$ B. Thus, as we have already pointed out, there is a certain interval, $v i z, 0<\mathrm{y}<\mathrm{B}$, in which the lower sign in (3.10), etc., corresponds to $r>a$. In this interval, the lower sigm solution corresponds to the low pressure side rather than the high pressure side of the shock wave, the veloc1ty $v_{1}$ being between $a_{1}$ and $a_{1}^{\prime}\left(c f_{0} 5.11\right.$ ). For any $v_{1}, y_{1}$ in this 1nterval, the high pressure solution $v_{3}, y_{3}$ can be found immediately from $(5.20)$, and for this high pressure solution we have (cf. $5.20, \mathrm{y}_{1}\left\langle B !\right.$ ) $\left.\mathrm{y}_{3}\right\rangle$ B and therefore $\nabla_{3}<a_{3^{\circ}}$. This proves the underscored statement above.

2. In par, 3 we have shown that there is, for any initial conditions, one and only one solution of the shock wave equations (3.10), (3.11) provided

$$
\frac{d T_{e}}{d \beta}>\frac{d T_{8}}{d \beta}
$$

on the high pressure side. Here $T_{8}$ is the temperature which belongs to a certain $\beta$ according to the shock wave theory, $T_{0}$ that which corresponds to the same $\beta$ in thermal equilibrium. In the notation of the present section, (5.29) is equivalent to

$$
\frac{d \beta^{\prime}}{d T}<\frac{d \beta}{d T}
$$

- 
Coimparing (5.18) with (5.19), it can eas1ly be seen that (5.30) is fulfilled when I> B, 1.e. always on the high pressure side of a shock wave, q.e.d.

Par. 6. The Temperature on the Hinh Pressure side.

In par. 5 we have found that the temperature increases continuously from the low pressure to the high pressure side if $v_{1}$ is only slightly greater than $a_{1}$, and this remains true if $\nabla_{1}$ becomes equal to a 80 that sharp shock wave front is formed. In the case $v_{1}=a_{a l}$, we have $T_{2}=T_{1}$, and a gradual increase from $T_{2}$ to $T_{3}$ on the high pressure olde of the wave front. On the other hand, for violent shock waves $\left(v_{1}>a_{1}\right)$, we have proved in (3.16c) that the temperature decreases from the wave front into the high pressure region as the inert degrees of freedom become excited. In this section we want to investigate where the limit between these two types of behavior is to be expected.

For th1s purpose we have to examine the dependence of $T$ on $\beta$ on the high pressure side. We know that $\beta$ incresses from the wave front into the high pressure region; therefore $\mathrm{dT} / \mathrm{d} \beta$ will be the quantity determining whether $\mathrm{T}$ increases or decreases. This derivative must, of course, be taken with the initial conditions (1.e. $\nabla$ and $c)$ kept fixed. The ralue of $\beta$ at which aT/a $\beta$ is to be calculated, must be chosen in the range of values occurring on the high pressure side. The lowest value of $\beta$ in that region, $\beta_{2}$, can be celculated from (4.2) and is given by

$$
\beta_{2}-\beta_{a}=\frac{T_{1}}{T_{2}}\left(\beta_{1}-\beta_{a}\right)
$$

The highest value, $\beta_{3}$, is in sufficlent approximation

$$
\beta_{3}=\beta_{1}+\frac{d \beta}{d \log T} \frac{T_{3}-T_{1}}{T_{1}}
$$

(6.2) is justified because, in the whole region in which $a r / d \beta>0$, the tempertature change $T_{3}-T_{1}$ 1s amall compared with $T_{1}$ 1tself. (cf. 6.1la, b). Therefore we cen also rewrite $(6.1)$ : 


$$
\beta_{2}=\beta_{1}-\left(\beta_{1}-\beta_{2}\right) \frac{T_{2}-T_{1}}{T_{1}}
$$

Both $(6.2)$ and $(6.10)$ are rather close to $\beta_{1}$ because $\beta_{1}-\beta_{2}$ and $\alpha \beta / d$ log $T$ are amall in practical cases; therefore it will be sufficient to calculate $a T / \alpha \beta$ for $\beta=\beta_{1}$ on the high pressure side.

More convenient that the explicit calculation of the derivative da/ab will be an investigation of the behavior of $T$ itself as a function of for given

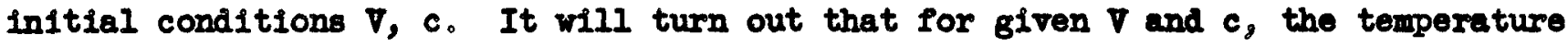
$T_{h}$ increases with $\beta$ for $\beta$ close to $\beta$ as defined in $(5.4)$, reeches a $\operatorname{maximum}$ for $\beta_{\mathrm{m}} \approx \beta_{0}+\frac{1}{4\left(\beta_{0}-\frac{1}{2}\right)}($ cr. 6.8$)$ and then decreases for lareor $\beta$. If, then, $\beta_{1}$ lies between $\beta_{0}$ and $\beta_{m}$, the temperature will increase from $T_{2}$ to $T_{3}$;' if $\beta_{1}$ is greater than $\beta_{\mathrm{m}^{2}}$ the temperature will decrease on the high pressure side. $\beta_{\mathrm{m}}$ is uniquely determined by $\nabla$ and $c$, therefore the condition $\beta_{1}<\beta_{\mathrm{m}}$ is equivalent to a condition for $v_{1} / a_{1}$ which will be given in $(6.14 c)$.

For the calculation, we insert $\nabla$ from $(3.10)$ into $(3.11)$ and obtain

$$
\frac{p}{\rho}=\frac{1}{(2 \beta-1)^{2}}\left[(2 \beta-1) c^{2}-\beta \nabla^{2} \mp \nabla \sqrt{\beta^{2} \nabla^{2}-(2 \beta-1) c^{2}}\right]
$$

Here we express $c^{2}$.in terms of $\beta_{0}$ by $(5.4)$, and introduce instead of $\beta$ and $\beta_{0}$

$$
b=\beta-\frac{1}{2} \quad b_{0}=\beta_{0}-\frac{1}{2}
$$

Then we obtain

$$
\frac{4 p}{p}=\frac{1}{b} \quad\left[\begin{array}{ll}
b_{0}+\frac{1}{0}+\frac{1}{b} & \sqrt{\left(b-b_{0}\right)\left(b-\frac{1}{4 b}\right)}
\end{array}\right]
$$

As usual, the lower sign is for the high pressure side of the shock ware. Putting $p /=$ Fr, and neglecting all powers of $b$ - $b_{0}$ higher than the square root, we obtain (5.7). 
For our present purpose, whall carry powers of $b$ - boup to the $3 / 2$ th, but we shall simplify the calculation by neglecting $1 / 4 b_{0}$ compared with $b_{0}$ Since the Interesting values of $b_{0}$ are at least 3 , we have $1 / 4 b^{2} \leqslant 1 / 36$.

Then (6.5) simplifies for the high pressure side to

$$
\frac{4 p}{p v^{2}}=\frac{b_{0}}{b}+\frac{\sqrt{b-b_{0}}}{b 3 / 2}
$$

(high pressure side). The maximum of the right hand side is obtained for

$$
\frac{1}{\sqrt{b-b_{0}}}\left(1-\frac{3\left(b-b_{0}\right)}{b}\right)=2 \frac{b_{0}}{b}
$$

In sufficient approximation, this equation is solved by

$$
b-b_{0}=\frac{1}{4 b+\frac{5}{b_{0}}}
$$

If the small terms of order $1 / b_{0}^{2}$ in $(6.5)$ are taken into account, $(6.6 \mathrm{~b})$ is reo placed by

$$
b-b_{0}=\beta-\beta_{0}=\frac{1}{4 b_{0}-\frac{1}{b_{0}}}=\frac{\beta_{0}-\frac{1}{2}}{4 \beta_{0}\left(\beta_{0}-1\right)}
$$

For $b_{0}=3$, this gives $b=b_{0}=\frac{3}{35}=0.086$. The maximum temperature is thus obtained at a value of $\beta$ which 18 only 81 ightly greater than $\beta$. For larger $\beta$, the factor $1 / \mathrm{b}$ in (6.5) has a stronger influence than the increase of the square root, so thet the temperature $(6.5)$ decreases again.

Thus we find that the temperature can increase on the high prossure side only if

$$
\beta_{1}<\beta_{m}=\beta_{0}+\frac{\beta_{0}-\frac{1}{2}}{\beta_{0}\left(\beta_{0}-1\right)}
$$


since $\beta_{0}$ is determined by $\nabla$ and $c(c f .5 .4),(6.8)$ is equivalent to a condition for $\nabla_{1} / a_{1}$. To derive this condition, we determine the dependence of $v$ on $\beta$ for fired $v$ and $c$. We have (cf.3.10)

$$
\frac{v}{v}=\frac{1}{2 \beta-1}\left[\beta \pm \sqrt{\beta^{2}-\frac{2 \beta-1}{2 \beta_{0}-1} \beta_{0}^{2}}\right]
$$

Introducing $b$, this gives

$$
\frac{2 v}{V}=1+\frac{1}{2 b} \pm \frac{1}{b} \sqrt{\left(b-\frac{1}{4 b}\right)\left(b-b_{0}\right)}
$$

For the negative sign (high pressure side), $(6,9 a)$ gives a monotonic decrease of $v$ with increasing $b$. Inserting in $(6.9 a)$ the value $\beta_{m}(c 9.6 .8)$ for $\beta=b+\frac{1}{2}$, we find on the high pressure side

$$
\nabla_{\operatorname{mh}}=\frac{1}{2} \nabla
$$

on the low pressure side

$$
\nabla_{m_{1}}=\frac{1}{2} v\left(1+\frac{1}{\beta-\frac{1}{2}}\right)
$$

Similarly, inserting $\beta_{\mathrm{m}}$ into the expression $(6.5)$ for the temperature, we obtain on the high pressure side

$$
R T_{\text {ma }}=[\mathrm{g}]_{\operatorname{mh}}=\frac{1}{4} \mathrm{v}^{2}
$$

on the low pressure side

$$
R T_{m_{1}}=\left[\begin{array}{l}
\not{D} \\
\hat{\rho}
\end{array}\right]_{m_{1}}=\frac{1}{4} v^{2} \quad\left(1-\frac{1}{b^{2}}\right) \approx \frac{1}{4} v^{2}\left(1-\frac{1}{\beta^{2}}\right)
$$


$7 \dot{5}$

Thus, at the value $\beta=\beta_{m}$ at which $T_{h}$ reaches its $\operatorname{maximum}$ for given $\nabla$ and $c$, we have the simple relation (cf。6.10a, ila)

$$
\nabla_{m b}=\sqrt{n_{m b}}=\frac{1}{2} \nabla
$$

and from (3.8b)

$$
p_{\operatorname{mh}}=\frac{1}{2} m \nabla=\frac{1}{2} P_{\max }
$$

where $P_{\max }$ is the pressure corresponding to $v=0$ 。

The velocity of sound $a^{\gamma}$ as defined in $(5.11)$ is then for $\beta=\beta_{\mathrm{m}}$ (ct. $6.118, b)$

$$
\begin{aligned}
& a_{m h}^{\prime}=\frac{1}{2} \nabla \sqrt{\frac{\beta}{\beta-1}} \\
& a_{m_{1}}^{B}=\frac{1}{2} \nabla \sqrt{\frac{\beta+1}{\beta}}
\end{aligned}
$$

It can easily be seen (cF, 5.12b) that in all practical cases aim - an is small compared with $v_{m}=a_{m}^{0}$ on both sides of the shock wave. Therefore we can identify $(6.13 a, b)$ with the actual velocity of sound a and obtain:

$$
\begin{gathered}
\frac{v_{m h}}{a_{m h}}=\sqrt{\frac{\beta-1}{\beta}} \approx 1-\frac{1}{2 \beta}-\frac{1}{8 \beta^{2}}+\ldots \\
\frac{v_{1}}{a_{m_{1}}}=1+\frac{1}{2 \beta}+\frac{3}{8 \beta^{2}}+\ldots
\end{gathered}
$$

Therefore we find that the temperature will increase on the high pressure if if

$$
\frac{v_{1}}{a_{1}}<\frac{v_{m_{1}}}{a_{m_{1}}}=1+\frac{1}{2 \beta}+\frac{3}{8 \beta^{2}}+\therefore \therefore
$$

(6.240) 
For $\beta=7 / 2$, the right hand side 1s 1.173. This shows that a temperature increase from $T_{2}$ to $T_{3}$ is restricted to very soft shock waves.

The temperature change $T_{3}-T_{2}$ Itself can be calculated from (6.2), (6.2a):

$T_{3}-T_{2}=\frac{d T_{B}}{d \beta} \cdot\left(\beta_{3}-\beta_{2}\right)=\frac{d T_{8}}{d \beta} \cdot \frac{T_{3}-T_{1}}{T_{1}} \cdot\left[\frac{d \beta}{d-g T_{e}}+\beta_{1}-\beta_{a}\right]$

Here we have denoted, as in par. 3 , by $T_{8}$ the temperature corresponding to a givon $\beta$ according to the shock wave theory, and by $T_{e}$ thet corresponding to the same $\beta$ in thermal equilibrium. $T_{2}-T_{1}$ in $(6.1 a)$ bas been replaced by $T_{3}-T_{1}$ because it will be show (cf. 6.17) that $T_{3}-T_{2}$ is small of a higher order. From (6.6) wo have in sufficient approximation

$$
\frac{d \log T_{8}}{d B}=\frac{1}{b_{0} \frac{3}{2}} \quad\left[\frac{1}{2 \sqrt{b_{1}-b_{0}}}-\sqrt{b_{0}}\right]
$$

Ilkewlse from $(6.6)$, we can calculate the difference between the high pressure and the low pressure value of $T$ for the same value of $\beta, \nabla 1 z, \beta$; this 18

$$
\frac{T_{3}-T_{1}}{T_{1}}=2 \frac{b_{1}-b_{0}}{b_{0} \frac{3}{2}}
$$

Inserting in (6.16), (6.16a) into $(6.15)$ and using the abbreviations $A$ and $B$ (ce. $5.15,16$ ) we obtain

$$
\frac{T_{3}-T_{2}}{T_{3}}=2(A+B)\left[1-\sqrt{4 b_{0}\left(b_{1}-b_{0}\right)}\right]
$$

which may also be written (cf.1.19) 


$$
\frac{T_{3}-T_{2}}{T_{3}}=\frac{c_{11}}{R\left(\beta_{1}-\frac{1}{2}\right) 3} \quad\left[1-\sqrt{4\left(\beta_{1}-\frac{1}{2}\right)\left(\beta_{1}-\beta_{0}\right)}\right]
$$

where $c_{1}$ is the specific heat of the inert degrees of freedom. From (6.17) It follows that the temperature change $T_{3}-T_{2}$ is greatest when $\beta_{1}=\beta_{0}, 1_{0} \theta_{0}$, for the softest shock waves; of course, this holds only when an actual wave front exists ( $1_{0} \theta_{0}$, for $v_{1}>a_{a 1}$ ) because otherwise $T_{2}$ cannot be defined. For "harder" shock waves, $1 . \theta_{0}$, greater $\nabla_{1} / a_{1}$ and $\beta_{1}-\beta_{0}$, the square bracket decreases and reaches zero for $\beta_{1}=\beta_{m}(c F, 6,8)$ as must be expected. For air of inftial temperature $T_{1}=300^{\circ}$, we have $c_{11}=0.007 R$; then from $(6.17 a)$ the maximum pose1ble value of $T_{3}-T_{2}$ is $0.00025 T_{3}=0.08^{\circ}$ 。 The temperature increase $T_{3}-T_{2}$, If 1 occurs at all, is therefore extremely small in air at normal temperature. The temperature difference $T_{m h}-T_{m}$, on the other hand, is appreciable, $v i z$ (cr.6.11) $1 / 9 T_{1}=33^{\circ}$ )

Finally, the developments of this section can be used to justify those of par,5. In that section, wo heve neglected in (6.5) and similar equations all powers of $b$ - $b_{0}$ higher than the square root。 This lo justifled as long as $\beta-\beta_{0}$ Is emall compared with $\beta_{m}-\beta_{0}$ as given by $(6,8)$. How the largest value of $\beta-\beta_{0}$ which we have used in par. 5 is obtained for $y=2 B+A$ (cP.5.15, 16). Using $(5.8),(6.8)$ and neglecting quantities of relative order $1 / \beta^{2}$ we have.

$$
\sqrt{\frac{\left(\beta-\beta_{0}\right)_{\max }}{\beta_{m} \beta_{0}}}=2 b_{0}^{2}(2 B+A)=\frac{2 d \beta / d \log T+\beta-\beta_{a}}{\beta_{0}-\frac{1}{2}}
$$

For air at $300^{\circ}$, this is about 0.005 . 
MASTER

MASTER:

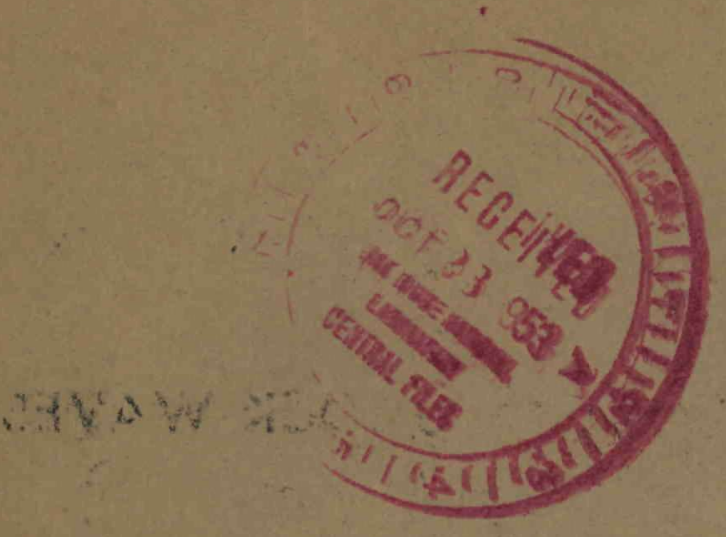

MASTER

MASTER 